\title{
The ESO UVES advanced data products quasar sample
}

\section{Cosmological evolution of the neutral gas mass density $\star$}

\author{
T. Zafar $^{1}$, C. Péroux ${ }^{1}$, A. Popping ${ }^{2}$, B. Milliard ${ }^{1}$, J.-M. Deharveng ${ }^{1}$, and S. Frank ${ }^{1,3}$ \\ 1 Aix Marseille Université, CNRS, LAM (Laboratoire d'Astrophysique de Marseille) UMR 7326, 13388 Marseille, France \\ e-mail: tayyaba.zafar@oamp.fr \\ 2 International Centre for Radio Astronomy Research (ICRAR), The University of Western Australia, 35 Stirling Hwy, \\ WA 6009 Crawley, Australia \\ ${ }^{3}$ Department of Astronomy, Ohio State University, 140 West 18th Avenue, Columbus, OH 43210-1173, USA
}

Received 23 January 2013 / Accepted 1 July 2013

\begin{abstract}
Damped absorbers, seen in the spectra of background quasars, are unique probes to select H I-rich galaxies. These galaxies allow one to estimate the neutral gas mass over cosmological scales. The neutral gas mass is a possible indicator of gas consumption as star formation proceeds. The damped Ly $\alpha$ absorbers (DLAs; $N_{\mathrm{HI}} \geq 2 \times 10^{20} \mathrm{~cm}^{-2}$ ) and sub-DLAs $\left(10^{19} \leq N_{\mathrm{HI}} \leq 2 \times 10^{20} \mathrm{~cm}^{-2}\right)$ are believed to contain a large fraction of neutral gas mass in the Universe. In Paper I of the series, we presented the results of a search for DLAs and sub-DLAs in the European Southern Observatory (ESO) Ultraviolet Visual Echelle Spectrograph (UVES) advanced data products dataset of 250 quasars. Here we use an unbiased subsample of sub-DLAs from this dataset to derive their statistical properties. We built a subset of 122 quasars ranging from $1.5<z_{\mathrm{em}}<5.0$, suitable for statistical analysis. The statistical sample was analyzed in conjunction with other sub-DLA samples from the literature. This resulted in a combined sample of 89 sub-DLAs over a redshift path of $\Delta z=193$. We derived the redshift evolution of the number density and the line density for sub-DLAs and compared them with the Lyman-limit systems (LLSs) and DLA measurements from the literature. The results indicate that these three classes of absorbers are evolving in the redshift interval $1.0<z<5$.0. Thanks to the ESO UVES advanced data products data, we were able to determine the column density distribution, $f_{\mathrm{HI}}(N, z)$, down to the sub-DLA limit. The flattening of $f_{\mathrm{HI}}(N, z)$ in the sub-DLA regime is present in the observations. The redshift evolution of $f_{\mathrm{HI}}(N, z)$ down to $\log N_{\mathrm{HI}}=19.0 \mathrm{~cm}^{-2}$ is also presented, indicating that there are more sub-DLAs at high redshift than at low redshift. $f_{\mathrm{HI}}(N, z)$ was also used to determine the neutral gas mass density, $\Omega_{\mathrm{g}}$, at $1.5<z<5.0$. The complete sample shows that sub-DLAs contribute $8-20 \%$ to the total $\Omega_{\mathrm{g}}$ from $1.5<z<5.0$. In agreement with previous studies, no evolution of $\Omega_{\mathrm{g}}$ was observed from low to high redshift (i.e., $1.5<z<5.0$ ), suggesting that star formation alone cannot explain this non-evolution and replenishment of gas and that recombination of ionized gas is needed.
\end{abstract}

Key words. galaxies: abundances - galaxies: evolution - galaxies: high-redshift - quasars: absorption lines - quasars: general

\section{Introduction}

Baryons account for only a small fraction of mass in the Universe (Fukugita \& Peebles 2004). In neutral and molecular phase they are reservoirs of gas from which stars form over cosmological scales. A way of measuring the assembly of galaxies is to probe the rate at which they convert their gas into stars. Therefore, to understand this, it is important to determine the cosmological mass density of neutral gas $\left(\Omega_{\mathrm{g}}\right.$; Ma $\&$ Bertschinger 1994; Klypin et al. 1995). The observations of H I-rich galaxies, selected by the imprint they leave on a background quasar, allow one to measure the neutral gas mass density, $\Omega_{\mathrm{g}}$. The quasar absorbers are divided into several classes according to the number of atoms along the line of sight: the damped Ly $\alpha$ absorbers (DLAs) with $N_{\mathrm{HI}} \geq 2 \times 10^{20} \mathrm{~cm}^{-2}$, the sub-DLAs with $10^{19} \leq N_{\mathrm{HI}} \leq 2 \times 10^{20} \mathrm{~cm}^{-2}$, the Lyman-limit systems (LLSs) with $1.6 \times 10^{17} \leq N_{\mathrm{HI}} \leq 10^{19} \mathrm{~cm}^{-2}$ and the Ly $\alpha$ forest with $N_{\mathrm{HI}} \leq 1.6 \times 10^{17} \mathrm{~cm}^{-2}$.

Ly $\alpha$ absorbers provide a useful probe of the distribution of baryonic matter in the Universe over cosmological scales. The

* Table 1 and Appendix are available in electronic form at http://www . aanda.org
$\mathrm{H}$ I column density distribution function, $f_{\mathrm{HI}}(N, z)$, for different classes of absorbers is determined by counting the number of Ly $\alpha$ absorbers in the spectra of background quasars (e.g., Wolfe et al. 1995; Storrie-Lombardi \& Wolfe 2000; Prochaska et al. 2005; Noterdaeme et al. 2009, 2012). Current measurements of the $f_{\mathrm{HI}}(N, z)$ distribution span almost ten orders of magnitude in column density, ranging from $N_{\mathrm{HI}} \sim 10^{12}-10^{22}$. The $f_{\mathrm{HI}}(N, z)$ distribution for quasar absorbers is analogous to the luminosity function of galaxy surveys. This function is then used to derive the amount of neutral gas mass as a function of redshift.

The contribution of sub-DLAs to the total neutral gas mass is the source of intense discussion in recent years (see Viegas 1995; Péroux et al. 2005; Guimarães et al. 2009). The contribution of sub-DLAs cannot be neglected in an accurate determination of the total neutral gas mass. The gas in LLSs/sub-DLAs is located in extended haloes, whereas the gas in DLAs is located in dense and compact regions (Péroux et al. 2003b; van de Voort et al. 2012). The evolution of $\Omega_{\mathrm{g}}$ has been open to debate (Péroux et al. 2005; Zwaan et al. 2005a; Rao et al. 2006; Lah et al. 2007; Noterdaeme et al. 2009; Martin et al. 2010; Noterdaeme et al. 2012; Braun 2012). At $z=0$, the neutral gas is traced by the hyperfine $21-\mathrm{cm}$ emission of atomic hydrogen, but the limited 
sensitivity of current generation of radio telescopes prevents detection beyond $z \sim 0.2$ (Lah et al. 2007; see also Chang et al. 2010).

In this work we take advantage of the advanced data products archival data of UVES (Dekker et al. 2000) mounted at the ESO-Very Large Telescope (VLT). The excellent spectral resolution of UVES allows measuring of the H I column density with high precision. In Zafar et al. (2013, hereafter Paper I), the details of the ESO UVES advanced data products dataset together with the new $N_{\mathrm{HI}}$ measurements of DLAs/sub-DLAs have been provided. Because the database is not a blind search for subDLAs, we build here an unbiased statistical sample. Our main aim is to include absorbers down to $\log N_{\mathrm{HI}} \geq 19.0$ to determine the shape of the $f_{\mathrm{HI}}(N, z)$ in the sub-DLA regime. The current sample allows us to determine the $f_{\mathrm{HI}}(N, z)$ redshift evolution. Quantities such as the number density and line density for different classes of quasar absorbers are also determined. Moreover, the incidence of the sub-DLAs together with the DLAs are used to determine the total gas mass density.

In Sect. 2 we define our dataset. The "statistical" and combined samples are defined in Sects. 3 and 4. In Sect. 5 we present our results for $f_{\mathrm{HI}}(N, z)$ and $\Omega_{\mathrm{g}}$, followed by a discussion. Conclusions are provided in Sect. 6. All log values and expressions correspond to $\log$ base 10. Throughout the paper we adopt the $\Lambda \mathrm{CDM}$ cosmology with the cosmological parameters $\Omega_{\Lambda}=0.7, \Omega_{\mathrm{m}}=0.3$, and $H_{0}=70 \mathrm{~km} \mathrm{~s}^{-1} \mathrm{Mpc}^{-1}$ (e.g., Spergel et al. 2003).

\section{EUADP sample}

Details of the building of the quasar sample can be found in Paper I and are briefly summarized here. The sample consists of 250 quasar spectra ranging from $0.191 \leq z_{\mathrm{em}} \leq 6.311$ taken with the VLT instrument UVES. The lowest resolving power of the UVES is $\sim 41400$ when a slit of $1^{\prime \prime}$ is used. The echelle spectrograph covers the wavelength range 300-500 $\mathrm{nm}$ (BLUE) and 420-1100 nm (RED) by use of different standard and nonstandard settings. The data were downloaded from the ESO UVES advanced data products (EUADP) facility. The downloaded data were processed by the ESO UVES pipeline (version 3.2) within the MIDAS environment with the best available calibration data. The different spectra of each quasar were then merged after interpolating to a common frame. Throughout the paper, this sample of quasars is coined the "EUADP" sample.

To derive a complete census of DLAs/sub-DLAs, an automated search accompanied by a visual search was made to search for DLAs and sub-DLAs. The algorithm consists of building an equivalent-width spectrum over 400-pixel-wide boxes for each quasar blueward of the Ly $\alpha$ emission line of the quasar (see Paper I). This search resulted in 93 DLAs and 57 sub-DLAs whose Ly $\alpha$ absorption lines are covered by our data. A careful search in the literature indicated that 87 of these DLAs and 44 of these sub-DLAs have been reported before. The $N_{\mathrm{HI}}$ measurements for the remaining 6 DLAs and 13 sub-DLAs are reported in Paper I. These DLA/sub-DLAs are confirmed by the detection of lines from higher-order Lyman series and associated metal-lines. Their H I column densities were determined using Voigt-profile fitting with FITLYMAN package in MIDAS (Fontana \& Ballester 1995). In addition, another 47 DLAs/subDLAs along the lines of sight of EUADP quasars have been found for which $\operatorname{Ly} \alpha$ absorption lines are not covered by our data. This yields a total of 197 DLAs/sub-DLAs along the linesof-sight of 250 EUADP quasars.

\section{Defining the statistical EUADP sample}

The EUADP data originally come from different observing programs where quasars were targeted by several groups for a variety of studies with different goals, many of which include the analysis of a known DLA. We have followed a strict and careful process to select quasar lines-of-sight and sub-DLAs that can be used for statistical analysis. This process, detailed below, led to a subset of the EUADP sample dubbed the "statistical" EUADP sample.

\subsection{Survey sensitivity for the statistical EUADP sample}

The first step was defining the redshift path for each quasar. As common is practice, we defined a redshift interval $\Delta z=$ $z_{\max }-z_{\min }$ to obtain the redshift path over which we could reliably determine the presence of damped absorbers. The lowest redshift $\left(z_{\min }\right)$ is either the lowest redshift at which the signalto-noise $(\mathrm{S} / \mathrm{N})$ ratio ensures the detection of absorption features at the sub-DLA threshold of $E W_{\text {rest }}=2.5 \AA$, or where an intervening LLS is present and thus prohibits the detection of any absorption feature at wavelengths shorter than the restframe Lyman break ( $912 \AA$ ). The higher redshift value of the two is adopted as $z_{\min }$ of the quasar. The highest redshift $\left(z_{\max }\right)$ is $3000 \mathrm{~km} \mathrm{~s}^{-1}$ blueward of the quasar emission redshift. In addition, some of the UVES-combined spectra have distinct spectral gaps due to no-overlapping settings, which were excluded from the redshift path calculation. The resulting values are provided for each line of sight in Table 1.

Furthermore, we ignored quasars with $z_{\mathrm{em}}<1.51$ because the UVES spectral coverage does not extend blueward to wavelengths required for detecting the $\operatorname{Ly} \alpha$ line. As a result of the $\mathrm{S} / \mathrm{N}$ cut, the quasars above $z_{\mathrm{em}}>5.0$ were excluded because the density of absorbers becomes high enough beyond that redshift to affect our ability to reliably determine the absence or presence of sub-DLAs. In addition, we examined the abstract of each observing proposal to determine if any knowledge of the sightline characteristics were known prior to the execution of the proposal that may represent a bias. We excluded all sightlines that were observed for studying the $\operatorname{Ly} \alpha$ forest and the intergalactic medium (IGM). This includes He II Gunn-Peterson effect, low column density distribution, and the tomography of the IGM as traced by quasar pairs or groups. Indeed, these sightlines might be biased toward having no high $N_{\mathrm{HI}}$ system, precisely the type of feature we are looking for in the present study. We chose the conservative approach to exclude all these redshift paths and sub-DLAs from our analysis. Broad-absorption-line (BALs) quasars are often excluded from the search for quasar absorbers. Indeed, the gas associated with the quasar itself can be confused with DLAs. The EUADP sample contains a few BAL quasars, but the absorption features are weak and cannot be confused with DLAs. In the appendix, we describe the reason for including or excluding each individual $z_{\mathrm{em}}>1.5$ quasar sightline and sub-DLA. This leaves us with a statistical EUADP sample of 122 quasars from the original 250 EUADP sample (see Table 2).

Where the archival quasar spectra were targeted for the study of dark-matter halos, black-hole masses, stellar masses, and quasar luminosities. where an absorber is not the interest of the study, we included the full redshift path. A large percentage $(\sim 45 \%)$ of the quasars in the EUADP sample were targeted because there was a DLA along their line of sight (studies for deuterium, molecules, or metal abundances), though many lines of sight have been targeted for a specific known DLA 
Table 2. Summary of the number of quasars and sub-DLAs for samples included in this study.

\begin{tabular}{|c|c|c|c|c|}
\hline $\begin{array}{l}\text { Sample } \\
\text { description }\end{array}$ & Ref. & $\Delta z$ & $\begin{array}{l}\text { QSOs } \\
\text { included }\end{array}$ & $\begin{array}{l}\text { Sub-DLAs } \\
\text { included }\end{array}$ \\
\hline$\overline{\text { EUADP }}$ & Paper I & $\cdots$ & 250 & 57 \\
\hline Statistical EUADP & This work & 88.2 & 122 & 27 \\
\hline Previous sub-DLA & $\begin{array}{l}\text { Péroux et al. (2003a, 2005) } \\
\text { O'Meara et al. (2007) }\end{array}$ & 104.9 & 116 & 62 \\
\hline Combined sub-DLA & This work & 193.1 & 238 & 89 \\
\hline
\end{tabular}

Notes. We used a subset of the EUADP sample, dubbed the statistical EUADP sample, combined with previous sub-DLA studies.

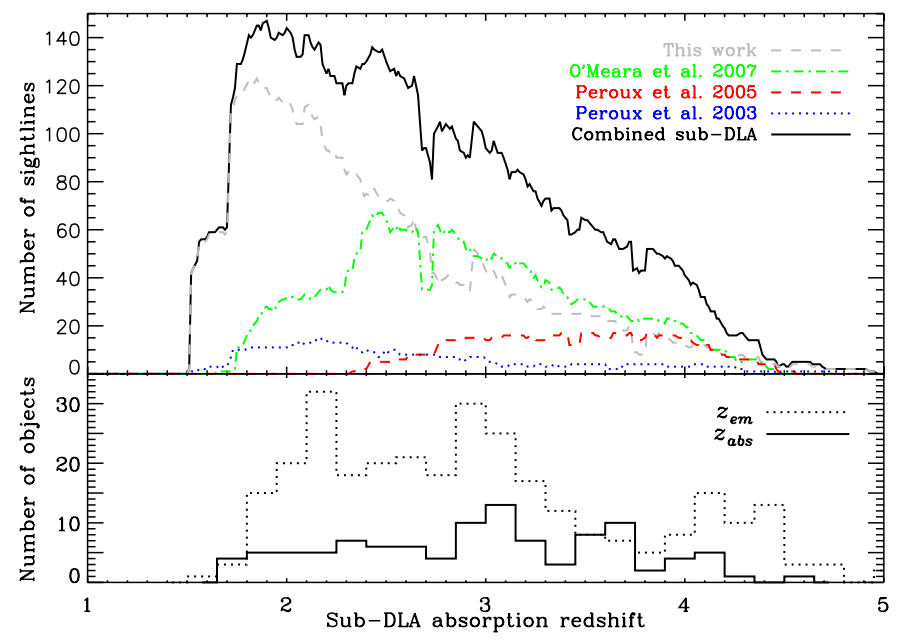

Fig. 1. Upper panel: redshift sensitivity function, $g(z)$, as a function of sub-DLA redshift for the statistical EUADP (gray), O'Meara et al. (2007) MIKE + ESI (green), Péroux et al. (2005, red), Péroux et al. (2003a, blue), and combined sub-DLA sample (black). Lower panel: the distribution of quasar emission and absorption redshift for the combined sub-DLA sample is illustrated by the black dotted and black solid lines, respectively.

without taking into consideration a sub-DLA along the same line of sight. When an archival quasar spectra was targeted for studying a DLA/sub-DLA, we removed the Ly $\alpha$ regions of a few angstroms in the trough (according to the column densities) from the redshift path, and the $\operatorname{Ly} \beta$ regions for DLAs with $\log N_{\mathrm{HI}} \gtrsim 20.90$. The working hypothesis was that the presence of a targeted DLA/sub-DLA does not effect the incidence of other systems. This methodology follows the one applied by others in the statistical analysis of archival data (Péroux et al. 2003a; Ribaudo et al. 2011).

The cumulative number of lines of sight along which an absorber at any given redshift could have been detected in Ly $\alpha$, i.e., redshift sensitivity $g(z)$, is defined as:

$g(z)=\sum H\left(z_{i}^{\max }-z\right) H\left(z-z_{i}^{\min }\right)$,

where $H$ is the Heaviside step function. The plot of $g(z)$ versus the sub-DLA redshift for the statistical EUADP sample is shown in Fig. 1. The sharp feature in $g(z)$ at $z \approx 2.82$ is caused by a spectral gap between the BLUE 390 and RED 580 settings. Another feature at $z \approx 3.8$ is due to a spectral gap in the range of $5756-5834 \AA$ in the RED 580 setting. The total redshift path of our statistical EUADP sample is $\Delta z=88.2$. For a comparison, the Magellan Inamori Kyocera Echelle (MIKE) and the Echellette Spectrograph and Imager (ESI) sub-DLA sample

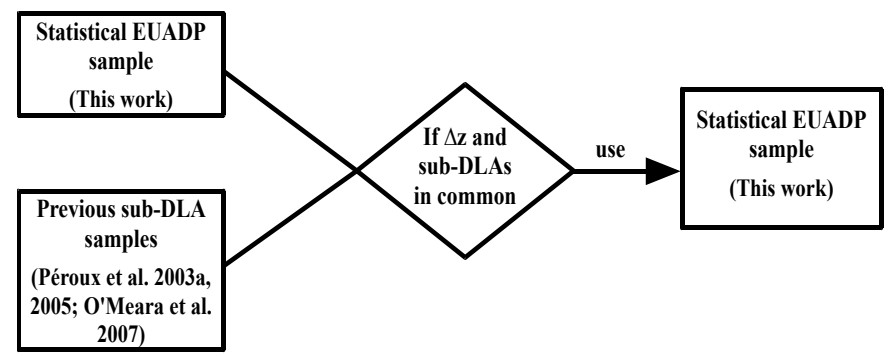

Fig. 2. Flowchart describing the building of the combined sub-DLA sample. For quasars in common with previous sub-DLA samples and the work presented here, we used $N_{\mathrm{HI}}$ and the redshift path from this work.

from O'Meara et al. (2007) and UVES samples from Péroux et al. (2003a, 2005) are also shown in Fig. 1. Their redshift paths are $\Delta z=91.8,16.6$, and 23.9 respectively. The EUADP sample includes a larger number of quasars at lower redshift than other samples.

\subsection{Absorber selection for the statistical EUADP sample}

We defined our statistical EUADP sample as the catalog of subDLAs of the EUADP sample from Paper I restricted to the redshift path defined above. The complete log of DLAs/sub-DLAs, redshifts, and column densities together with references are given in Table 1. Only sub-DLAs shown in boldface in Table 1 were included in the statistical EUADP sample. The resulting statistical EUADP sample consists of 27 sub-DLAs found by surveying the 122 quasars' lines of sight.

\section{Defining the combined sub-DLA sample}

For the remained of the paper, the statistical EUADP sample is used in combination with previous sub-DLA studies. For the analysis of sub-DLAs, we included the statistical EUADP sample and the results of the high-resolution surveys by Péroux et al. (2003a, 2005) and O'Meara et al. (2007) in our statistics. When combining these samples, we encountered cases where the same quasars were targeted in two independent surveys. For these we used $\Delta z$ and the sub-DLA from this work. For example, because the EUADP sample is based on UVES archival data, by construction it includes the data from Péroux et al. (2005) which are based on a PI UVES project aiming at finding new sub-DLAs at high redshift. The method used prevents any multiple counting in such cases. The procedure of defining the combined subDLA sample is illustrated in Fig. 2. In Fig. 1, $g(z)$ for the combined sub-DLA, Péroux et al. (2005), Péroux et al. (2003b), and O'Meara et al. (2007) samples are shown. The total redshift path for the combined sub-DLA sample is $\Delta z=193.1$.

The resulting combined sub-DLA sample consists of 89 subDLAs found by surveying 238 quasars (see Table 2). The column density distributions of the combined sub-DLA sample, Péroux et al. (2005), Péroux et al. (2003a), and MIKE + ESI samples from O'Meara et al. (2007) are shown in Fig. 3.

\section{Statistical sample properties}

\subsection{Number density}

The number density of quasar absorbers, a quantity directly observable, is the number of absorbers per unit redshift, i.e., 
Table 3. Summary of the number density $(\mathrm{d}(n) / \mathrm{d} z)$ and line number density $(\mathrm{d}(n) / \mathrm{d} X)$ of DLAs and sub-DLAs.

\begin{tabular}{|c|c|c|c|c|c|c|c|c|c|c|c|c|}
\hline \multirow{2}{*}{$\begin{array}{l}\log N_{\mathrm{HI}} \\
z \text { range }\end{array}$} & \multicolumn{6}{|c|}{$>19.0$} & \multicolumn{6}{|c|}{$>20.3$} \\
\hline & $\langle z\rangle$ & $\mathrm{d} z$ & $\mathrm{~d} X$ & \# & $\mathrm{d}(n) / \mathrm{d} z$ & $\mathrm{~d}(n) / \mathrm{d} X$ & $\langle z\rangle$ & $\mathrm{d} z$ & $\mathrm{~d} X$ & \# & $\mathrm{d}(n) / \mathrm{d} z$ & $\mathrm{~d}(n) / \mathrm{d} X$ \\
\hline $1.51-2.00$ & 1.80 & 29.9 & 87.3 & 4 & $0.35 \pm 0.12$ & $0.12 \pm 0.04$ & 1.84 & 95.6 & 279.4 & 19 & $0.22 \pm 0.05$ & $0.08 \pm 0.02$ \\
\hline 2.00 & 2.26 & 49.2 & 156.8 & 11 & $0.43 \pm 0.10$ & $0.14 \pm$ & 2.27 & 125.1 & 396.6 & 26 & $0.21 \pm 0.04$ & 0.07 \\
\hline $2.50-3.00$ & 2.76 & 47.1 & 162.8 & 24 & $0.72 \pm 0.13$ & $0.21 \pm 0.04$ & 2.73 & 87.2 & 301.0 & 18 & $0.21 \pm 0.05$ & $0.06 \pm 0.01$ \\
\hline $3.00-3.50$ & 3.21 & 33.7 & 124.5 & 23 & $0.96 \pm 0.18$ & $0.26 \pm 0.05$ & 3.25 & 64.8 & 239.6 & 18 & $0.28 \pm 0.07$ & $0.08 \pm 0.02$ \\
\hline $3.50-4.00$ & 3.72 & 23.3 & 91.7 & 19 & $1.20 \pm 0.24$ & $0.30 \pm 0.06$ & 3.77 & 49.5 & 194.6 & 19 & $0.38 \pm 0.09$ & $0.10 \pm 0.02$ \\
\hline $4.00-5.00$ & 4.18 & 9.9 & 41.0 & 8 & $1.25 \pm 0.38$ & $0.30 \pm 0.09$ & 4.20 & 23.1 & 95.1 & 10 & $0.43 \pm 0.14$ & $0.10 \pm 0.03$ \\
\hline $1.51-5.00$ & $\ldots$ & 193.1 & 664.1 & 89 & $\ldots$ & $\ldots$ & $\ldots$ & 445.4 & 1506.9 & $\ldots$ & $\ldots$ & $\ldots$ \\
\hline
\end{tabular}

Notes. The DLA results are taken from the Péroux et al. (2003b) sample. The $\mathrm{d}(n) / \mathrm{d} z$ and $\mathrm{d}(n) / \mathrm{d} X$ for sub-DLAs is measured from the contribution of both DLAs and sub-DLAs. The final row shows the total redshift path and absorption path length for both DLAs and sub-DLAs.

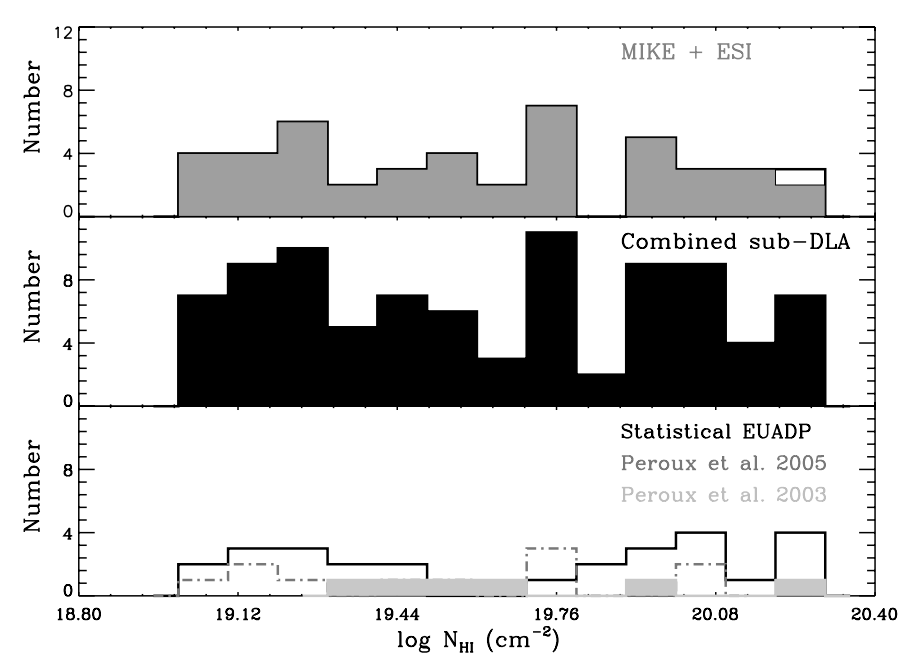

Fig. 3. Histograms showing the number of sub-DLAs as a function of $\log N_{\mathrm{HI}}$. Top panel: MIKE + ESI sample from O'Meara et al. (2007). The filled area represents the new sub-DLAs in MIKE + ESI sample and the unfilled area illustrates the ones overlapping with the combined sub-DLA sample. Middle panel: combined sub-DLA sample. Bottom panel: the solid black, dash-dotted gray and filled gray histograms show the contribution of sub-DLAs to the combined sub-DLA sample from the statistical EUADP, Péroux et al. (2005), and Péroux et al. (2003a) samples respectively.

$\mathrm{d}(n) / \mathrm{d} z$ also represented as $n(z)$,

$\mathrm{d}(n) / \mathrm{d} z=n(z)=\frac{m}{\mathrm{~d} z}$

where $m$ is the total number of absorbers in a given redshift bin and $\mathrm{d} z$ is computed by summing the redshift paths in this bin.

The combined sample allows one to determine the number density of sub-DLAs and compare it with those for the DLAs and LLSs from the literature. Figure 4 shows the number density per unit redshift for DLAs (Péroux et al. 2003b; Prochaska et al. 2005; Rao et al. 2006; Zwaan et al. 2005b), sub-DLAs (the combined sub-DLA sample), and LLSs (Songaila \& Cowie 2010; O'Meara et al. 2013). At low-redshift, results for DLAs come from the Rao et al. (2006) survey of quasars targeted because of known Mg II absorbers. The Zwaan et al. (2005b) results come from 21-cm emission of atomic hydrogen of $z=0$ analogues to DLAs. At low redshifts, the results of O'Meara et al. (2013) for LLS come from Hubble Space Telescope survey. The binning for our data was chosen with an increment of one half in redshift except for $4.0<z<5.0$ because of low number statistics. Vertical error bars reflect $1 \sigma$ Poisson uncertainty in the number of absorbers. All results are tabulated in Table 3.

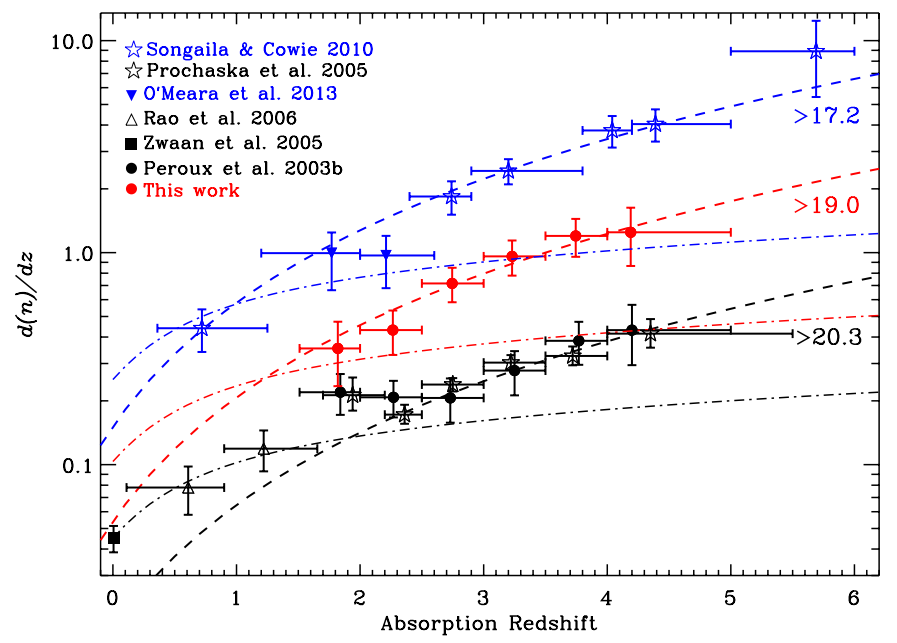

Fig. 4. Number density $(\mathrm{d}(n) / \mathrm{d} z)$ of quasar absorbers as a function of redshift. $\mathrm{d}(n) / \mathrm{d} z$ for DLAs: Péroux et al. (2003b, black circles), Prochaska et al. (2005, black open stars), Rao et al. (2006, black open triangles), and Zwaan et al. (2005b, black square) are shown. $\mathrm{d}(n) / \mathrm{d} z$ for sub-DLAs: the combined sub-DLA sample (red circles), and LLSs: Songaila \& Cowie (2010, blue open stars) and O'Meara et al. (2013, blue inverted triangles) are shown. The size of the horizontal bars represents the redshift bin sizes, and the vertical error bars are the $1 \sigma$ Poisson uncertainties in the number of absorbers. The blue dashed line is the simple evolution fit to the LLS from Songaila \& Cowie (2010). The red and black dashed lines are the LLS evolution fit scaled by a factor of 2.8 and 9.0 for the difference in $\mathrm{d}(n) / \mathrm{d} z$ of sub-DLAs and DLAs with respect to LLSs (in the range $2.5<z<3.0$ ), respectively. The black dot-dashed curve represents a non-evolving population of nonzero $\Lambda$-Universe. The red and blue dot-dashed curves are non-evolution curves scaled to a factor of 2.3 and 5.6, respectively (see text for more detail).

Songaila \& Cowie (2010) found that $\mathrm{d}(n) / \mathrm{d} z$ of LLSs can be well fitted by a simple evolution law of the form $\mathrm{d}(n) / \mathrm{d} z=$ $n_{3.5}[(1+z) / 4.5]^{\gamma}$ with $n_{3.5}=2.80 \pm 0.33$ and $\gamma=1.94_{-0.32}^{+0.36}$ for the entire redshift range from $0<z<6$, where $n_{3.5}$ is the value of $n(z)$ at $z=3.5$. In Fig. 4 we plot this simple evolution fit for LLS from Songaila \& Cowie (2010), which provides a reasonable fit to the data. For sub-DLAs and DLAs, the maximumlikelihood fit for the LLS was scaled according to a factor of difference in number density of the sub-DLA (by a factor of 2.8) and DLA (by a factor of 9.0) in the redshift range $2.5<z<3.0$. The redshift range was chosen arbitrarily. This shows that the number density of the absorbers per unit redshift is increasing with increasing redshift for all three classes of absorbers. The number density evolution inferred for these three classes 
T. Zafar et al.: Cosmological evolution of the neutral gas mass density

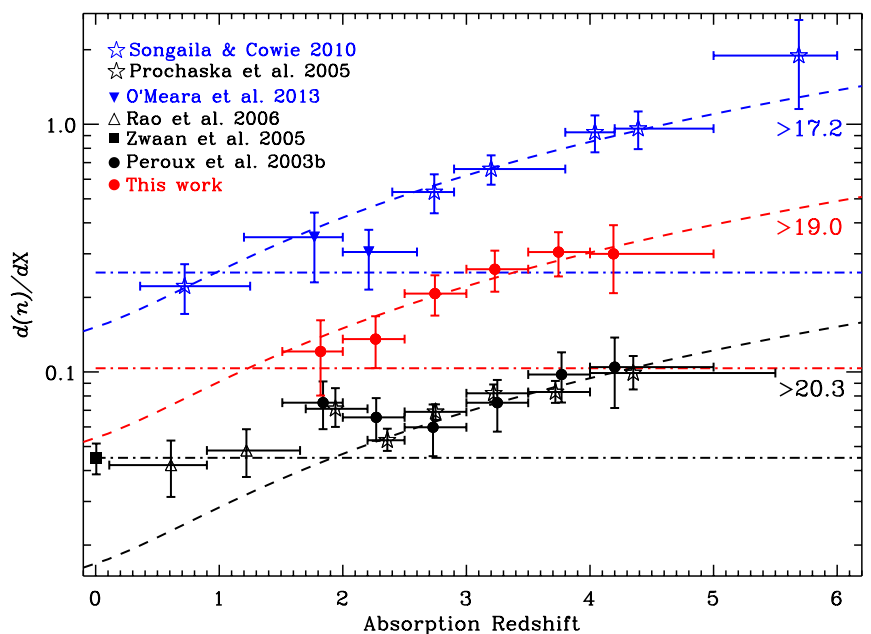

Fig. 5. Line density $(\mathrm{d}(n) / \mathrm{d} X)$ of quasar absorbers as a function of redshift. $\mathrm{d}(n) / \mathrm{d} X$ for DLAs, sub-DLAs and LLSs is shown as black, red, and blue data points, respectively. The symbols have the same meaning as in Fig. 4.

of quasar absorbers is generally steeper with increasing redshift (see Songaila \& Cowie 2010).

Assuming no-evolution in a non-zero $\Lambda$-Universe, the number density can be expressed as (see e.g., Péroux et al. 2005)

$n(z)=n_{0}(1+z)^{2}\left(\frac{H(z)}{H_{0}}\right)^{-1}$,

where $H(z)$ is the Hubble parameter given as

$H(z)=H_{0}\left[\Omega_{\mathrm{m}}(1+z)^{3}+\Omega_{\Lambda}\right]^{1 / 2}$.

As derived by Zwaan et al. (2005b), $n_{0}$ was taken to be $n(z=0)=0.045 \pm 0.006$. The non-evolution curve for DLAs is shown in Fig. 4. The non-evolution curve for LLS was scaled according to a factor of difference in number density of the LLS (5.6) in the redshift range $0.26<z<1.06$. For sub-DLAs, the non-evolution curve was arbitrarily scaled (by a factor of 2.3) in the middle of the DLA and LLS curves because there are no results available at low redshift. Departure from non-evolution beyond $z \sim 1$ is evident for the three classes of quasar absorbers. The DLAs seems to behave differently at $z<2$, which might be due to the different data surveys and methods.

\subsection{Line density}

The cosmological line density of quasar absorbers is the number of absorbers per unit absorption path length $\mathrm{d} X$ i.e., $\mathrm{d}(n) / \mathrm{d} X$. The line density of quasar absorbers, also termed $l(X)$, is the product of the comoving number density and the cross section of absorbers (see Prochaska et al. 2005). The values for quasar absorbers were calculated in the discrete limit by using

$\mathrm{d}(n) / \mathrm{d} X=l(X)=\frac{m}{\mathrm{~d} X}$.

This can also be expressed as $l(X) \mathrm{d} X=n(z) \mathrm{d} z$, where the absorption distance (Bahcall \& Peebles 1969) is defined as

$\mathrm{d} X=\int \frac{H_{0}}{H(z)}(1+z)^{2} \mathrm{~d} z$.

In Fig. 5 the line densities for DLAs (Péroux et al. 2003b; Prochaska et al. 2005; Rao et al. 2006; Zwaan et al. 2005b),

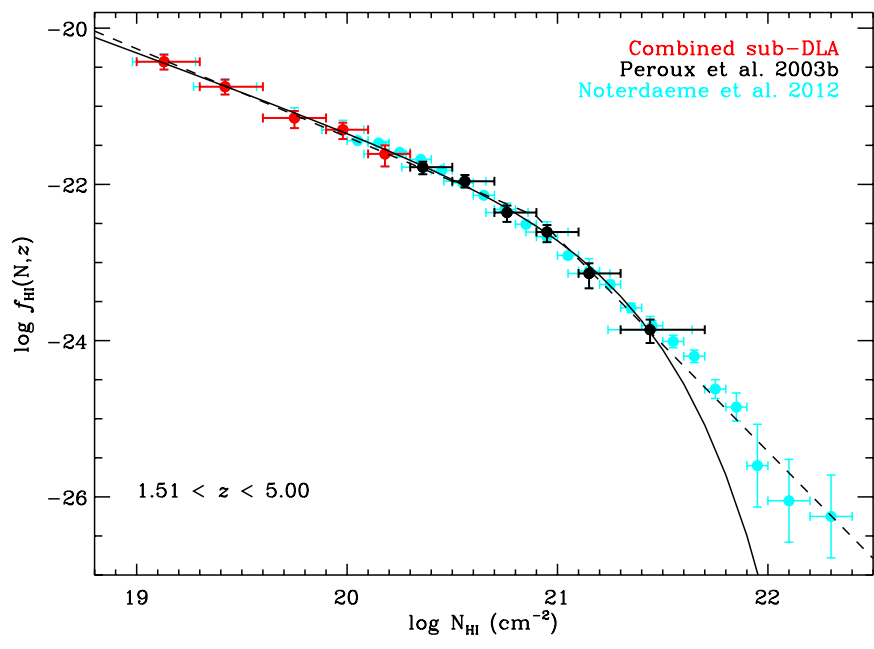

Fig. 6. $N_{\mathrm{HI}}$ frequency distribution of DLAs (Péroux et al. 2003b) and sub-DLAs (combined sub-DLA sample) in a range of $1.51<z<5.00$ is shown as black and red circles, respectively. The $\Gamma$-function and double power-law fit to these DLA and sub-DLA results are shown as solid and dashed black lines, respectively. The cyan data points represent the results from Noterdaeme et al. (2012) over two orders of magnitude in $N_{\mathrm{HI}}$. The departure at high column densities $\left(\log N_{\mathrm{HI}}>21.0\right)$ from the $\Gamma$-function shape is due to low number statistics at higher column density in the former studies.

sub-DLAs (combined sub-DLA sample), and LLSs (Songaila \& Cowie 2010; O'Meara et al. 2013) versus redshift are presented. The binning and error calculation procedure is the same as defined in Sect. 5.1. The results are tabulated in Table 3.

The simple evolution fit to the line density for the LLS from Songaila \& Cowie (2010) is also plotted in Fig. 5. For DLAs and sub-DLAs the fit is scaled in a similar manner as explained in Sect. 5.1. Clearly the line density for all three classes of absorbers increases with redshift. The rise in $\mathrm{d}(n) / \mathrm{d} X$ reflects an increase in the comoving number density or the cross-section of the absorber. In Fig. 5 the line density non-evolution curve for DLAs is shown and for LLSs and sub-DLAs is scaled in a similar manner as explained in Sect. 5.1. The flatness of the nonevolution curve reflects no variation in the covering fraction of absorbers on the sky with increasing redshift. There is a clear departure from non-evolution beyond $z \sim 1$ for all three classes of quasar absorbers.

\subsection{Column density distribution}

The distribution of $\mathrm{H} \mathrm{I}$ column densities for all classes of quasar absorbers has been investigated in several studies (e.g., Péroux et al. 2005; Ribaudo et al. 2011; Noterdaeme et al. 2012). The differential column density distribution, $f_{\mathrm{HI}}(N, z)$, describes the evolution of quasar absorbers as a function of atomic column density and redshift,

$f_{\mathrm{HI}}(N, z) \mathrm{d} N \mathrm{~d} X=\frac{m}{\Delta N \sum_{i=1}^{n} \Delta X_{i}} \mathrm{~d} N \mathrm{~d} X$,

where $m$ is the number of quasar absorbers in a range of column densities $(N, N+\mathrm{d} N)$ obtained from the observations of $n$ quasar spectra with the total absorption distance coverage $\sum_{i=1}^{n} \Delta X_{i}$. In Fig. 6 , we present the $f_{\mathrm{HI}}(N, z)$ of the combined sub-DLA sample while the values are tabulated in Table 4 . The horizontal bars indicate the bin sizes plotted at the mean column density for each bin, and the vertical error bars are Poissonian statistical errors. For a comparison with the DLA regime, the results 
Table 4. Summary of the column density distribution of the combined sub-DLA sample.

\begin{tabular}{lccc}
\hline \hline $\begin{array}{l}\log N_{\mathrm{HI}} \\
\text { range }\end{array}$ & $\#$ & $\begin{array}{c}\log \\
\left\langle N_{\mathrm{HI}}\right\rangle\end{array}$ & $\begin{array}{c}\log \\
f_{\mathrm{HI}}(N, z)\end{array}$ \\
\hline $19.00-19.30$ & 23 & 19.13 & $-20.43_{-0.10}^{+0.09}$ \\
$19.30-19.60$ & 21 & 19.42 & $-20.75_{-0.10}^{+0.09}$ \\
$19.60-19.90$ & 16 & 19.75 & $-21.15_{-0.13}^{+0.09}$ \\
$19.90-20.10$ & 18 & 20.06 & $-21.30_{-0.12}^{+0.09}$ \\
$20.10-20.30$ & 11 & 20.06 & $-21.61_{-0.16}^{+0.11}$ \\
\hline
\end{tabular}

of the Péroux et al. (2003b) samples are also shown with the same redshift bins. In recent SDSS samples, Noterdaeme et al. (2009, 2012) observed absorbers down to $\log N_{\mathrm{HI}}=20.0$. The results of Noterdaeme et al. (2012) are also plotted in Fig. 6. Empirically, $f_{\mathrm{H} \mathrm{I}}(N, z)$ were fitted by a power-law for various $N_{\mathrm{HI}}$ regimes (e.g., Tytler 1987; Rao et al. 2006; O’Meara et al. 2007). Early determinations of $f_{\mathrm{HI}}(N, z)$ for a broad column density range were defined with a single power-law (Tytler 1987), but the improved quality of observations shows that a single powerlaw does not represent the observations well over a broad column density range. For this reason, a $\Gamma$-function (power-law with an exponential turn-over), analogous to the Schechter function, was used to reasonably describe the observations (e.g., StorrieLombardi \& Wolfe 2000; Péroux et al. 2003b; Noterdaeme et al. 2009). In addition, the integral of this functional form used to compute $\Omega_{\mathrm{g}}$ converges, as opposed to a power law. The $\Gamma$-function is defined as

$f_{\mathrm{HI}}(N, z)=k_{\mathrm{g}}\left(\frac{N}{N_{\mathrm{g}}}\right)^{\alpha_{\mathrm{g}}} \mathrm{e}^{\left(-N / N_{\mathrm{g}}\right)}$.

We fit the data with the $\Gamma$-function for the total redshift range. The best-fit values of the parameters are provided in Table 5 together with the reduced $\chi^{2}\left(\chi_{v}^{2}\right)$ values that define the goodnessof-fit. We also fit the data with a double power-law resulting in the best-fit values of $k_{\mathrm{d}}=-22.41, N_{\mathrm{d}}=20.90$ (fixed), $\alpha_{\mathrm{d} 1}=-1.13$, and $\alpha_{\mathrm{d} 2}=-2.74$ (see also Noterdaeme et al. 2009). As apparent in Fig. 6, the column density distribution flattens in the sub-DLA regime. This flattening of the distribution above $\log N_{\mathrm{HI}}=18.0 \mathrm{~cm}^{-2}$ is indeed expected (e.g., Péroux et al. 2005; O'Meara et al. 2007; Guimarães et al. 2009). It is due to less self-shielding in the absorbers because at these column densities, part of the neutral gas of the absorber is ionized by incident UV flux (e.g., Katz et al. 1996; Pontzen et al. 2008; McQuinn et al. 2011; Altay et al. 2011). The distribution steepens around $\log N_{\mathrm{HI}}=20.3-21.5$ as the absorbing gas becomes fully neutral (Altay et al. 2011; Erkal et al. 2012) and because of the atomic-to-molecular hydrogen transition (Schaye 2001; Krumholz et al. 2009; Altay et al. 2011). There is a deficit of $\log N_{\text {H I }}>21.7$ DLAs in the Péroux et al. (2003b) sample because their redshift path is smaller than that of the SDSS surveys (Noterdaeme et al. 2009, 2012), the latter providing a better estimation of $f_{\mathrm{HI}}(N, z)$ down to $\log N_{\mathrm{HI}} \gtrsim 22$.

In a recent study, Erkal et al. (2012) argued that the atomicto-molecular hydrogen transitions do not significantly contribute to the high column density redshift-independent turnover. They furthermore argued that the similarity of the $f_{\mathrm{HI}}(N, z)$ at $z=3$ (e.g., Noterdaeme et al. 2009) and $z=0$ (Zwaan et al. 2005b) is due to the similarity of the highest central H I surface densities of high $-z$ and low- $z$ disks. van de Voort et al. (2012) advocated that above $\log N_{\mathrm{HI}}=17$ most of the absorbers reside inside haloes
Table 5. Best-fit parameters of the $\Gamma$-function fits (see Eq. [6]) to the $N_{\mathrm{HI}}$ frequency distribution for different redshift bins.

\begin{tabular}{lcccc}
\hline \hline$z$ range & $\log k_{\mathrm{g}}$ & $\log N_{\mathrm{g}}$ & $\alpha_{\mathrm{g}}$ & $\chi_{v}^{2}$ \\
\hline $1.51-5.00$ & -22.33 & 21.02 & -1.00 & 0.71 \\
$1.51-3.10$ & -22.30 & 21.08 & -0.95 & 1.16 \\
$3.10-5.00$ & -22.05 & 20.91 & -1.00 & 0.76 \\
\hline
\end{tabular}

Table 6. Summary of the column density distribution of the combined sub-DLA sample in two redshift bins.

\begin{tabular}{lcccccc}
\hline \hline \multirow{2}{*}{$\begin{array}{l}\text { range } \\
\log N_{\mathrm{HI}}\end{array}$} & \multicolumn{2}{c}{$1.51-3.10$} & \multicolumn{3}{c}{$3.10-5.00$} \\
range & $\#$ & $\left\langle N_{\mathrm{HI}}\right\rangle$ & $f_{\mathrm{HI}}(N, z)$ & $\#$ & $\left\langle N_{\mathrm{HI}}\right\rangle$ & $f_{\mathrm{HI}}(N, z)$ \\
\hline $19.00-19.40$ & 14 & 19.20 & $-20.64_{-0.13}^{+0.11}$ & 17 & 19.17 & $-20.29_{-0.12}^{+0.10}$ \\
$19.40-19.90$ & 14 & 19.60 & $-21.16_{-0.13}^{+0.10}$ & 15 & 19.65 & $-20.88_{-0.13}^{+0.10}$ \\
$19.90-20.30$ & 16 & 20.08 & $-21.48_{-0.12}^{+0.10}$ & 13 & 20.03 & $-21.30_{-0.14}^{+0.10}$ \\
\hline
\end{tabular}

and below $\log N_{\mathrm{HI}}=17$ the absorbers reside outside haloes. They also argued that the absorbing gas between column densities $17<\log N_{\mathrm{HI}}<21$ has never experienced a virial shock.

\subsection{1. $f_{\mathrm{HI}}(N, z)$ redshift evolution}

We estimated the column density distribution for low and high redshifts. The differential column density distributions for two redshift ranges, $1.51<z<3.1$ and $3.1<z<5.0$, are shown in Fig. 7. These redshift ranges are chosen to divide the number of sub-DLAs equally for each redshift bin. The results of the combined sub-DLA sample for two redshift bins are tabulated in Table 6. Because the $\mathrm{d} z$ were available to us, we fit the combined sub-DLA and Péroux et al. (2003b) results with the $\Gamma$-function for both redshift ranges; the best-fit values of the parameters are summarized in Table 5. For comparison, the column density distribution at $z=0$ is also shown in Fig. 7 from Zwaan et al. (2005b) and Braun (2012). The results of Zwaan et al. (2005b) come from the survey of $355 \mathrm{H} \mathrm{I} 21-\mathrm{cm}$ individual maps of nearby galaxies and the Braun (2012) results come from H I distribution, based on high-resolution maps of M31, M33, and the Large Magellanic Cloud (LMC). The data of Zwaan et al. (2005b) have a lower resolution and therefore cannot resolve the highest column densities. Braun (2012) used data at very highresolution and also applied a correction to the column density based on opacity. In Fig. 7, we overplotted the $\Gamma$-function fit from Zwaan et al. (2005b) with $\alpha_{\mathrm{g}}=1.24, \log k_{\mathrm{g}}=-22.91$ and $\log$ $N_{\mathrm{g}}=21.20$. We performed a $\Gamma$-function fit to the Braun (2012) results with $\alpha_{\mathrm{g}}=1.02, \log k_{\mathrm{g}}=-24.10$ and $\log N_{\mathrm{g}}=21.87$.

Figure 7 indicates that the $f_{\mathrm{HI}}(N, z)$ evolves with redshift. There is a tendency for a flattening of the distribution function in the sub-DLA regime at lower redshift, indicating that the number of sub-DLAs is larger at higher redshift. This evolution of subDLAs might be due to the merging history of absorbers or the ionization of the systems with time.

\subsubsection{Comparison with other studies}

We combined our study with previous studies to place constraints on the $f_{\mathrm{HI}}(N, z)$ over ten orders of magnitude in $N_{\mathrm{HI}}$. In Fig. 8, we show the column density distribution at $\langle z\rangle \sim 3$. In the DLA regime, the results come from Noterdaeme et al. (2012) 
T. Zafar et al.: Cosmological evolution of the neutral gas mass density
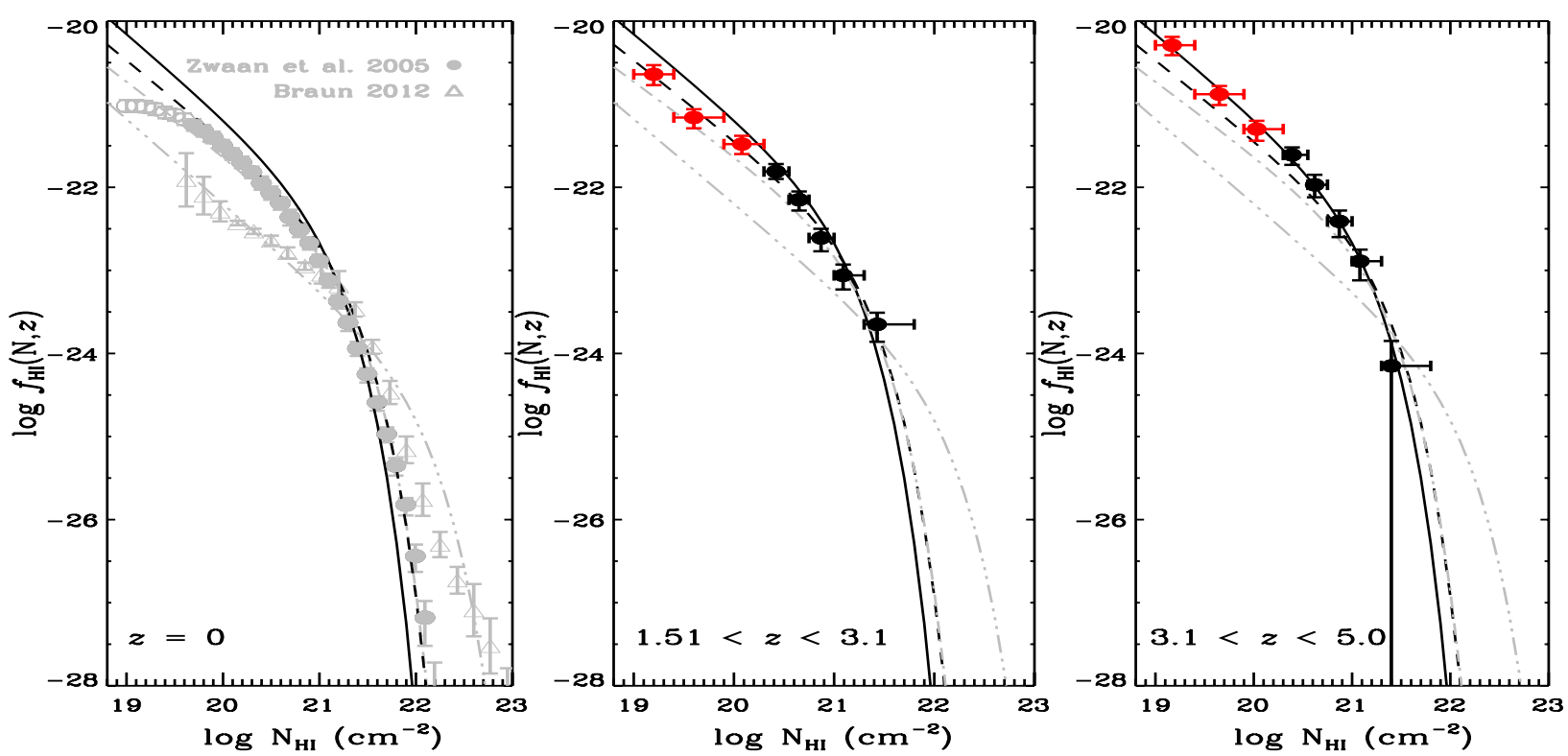

Fig. 7. Middle and right panels: differential column density distribution for two redshift ranges. The horizontal error bars are the bin sizes and the vertical error bars represent the Poissonian statistical errors on the column density distribution. The bins in red correspond to the combined sub-DLA sample results and bins in black correspond to the Péroux et al. (2003b) results. The black dashed and solid lines represent the $\Gamma$-function fit to the $f_{\mathrm{HI}}(N, z)$ in $3.1<z<5.0$ and $1.51<z<3.0$ redshift bins, respectively. Left panel: $f_{\mathrm{HI}}(N, z)$ at $z=0$ from Braun (2012, gray open triangles) and Zwaan et al. (2005b, gray filled circles). Open gray circles correspond to the measurements below the sensitivity limit of Zwaan et al. (2005b). The gray triple dot-dashed and dot-dashed line is $\Gamma$-function fit to Braun (2012) and Zwaan et al. (2005b) data, respectively, above their sensitivity limit.

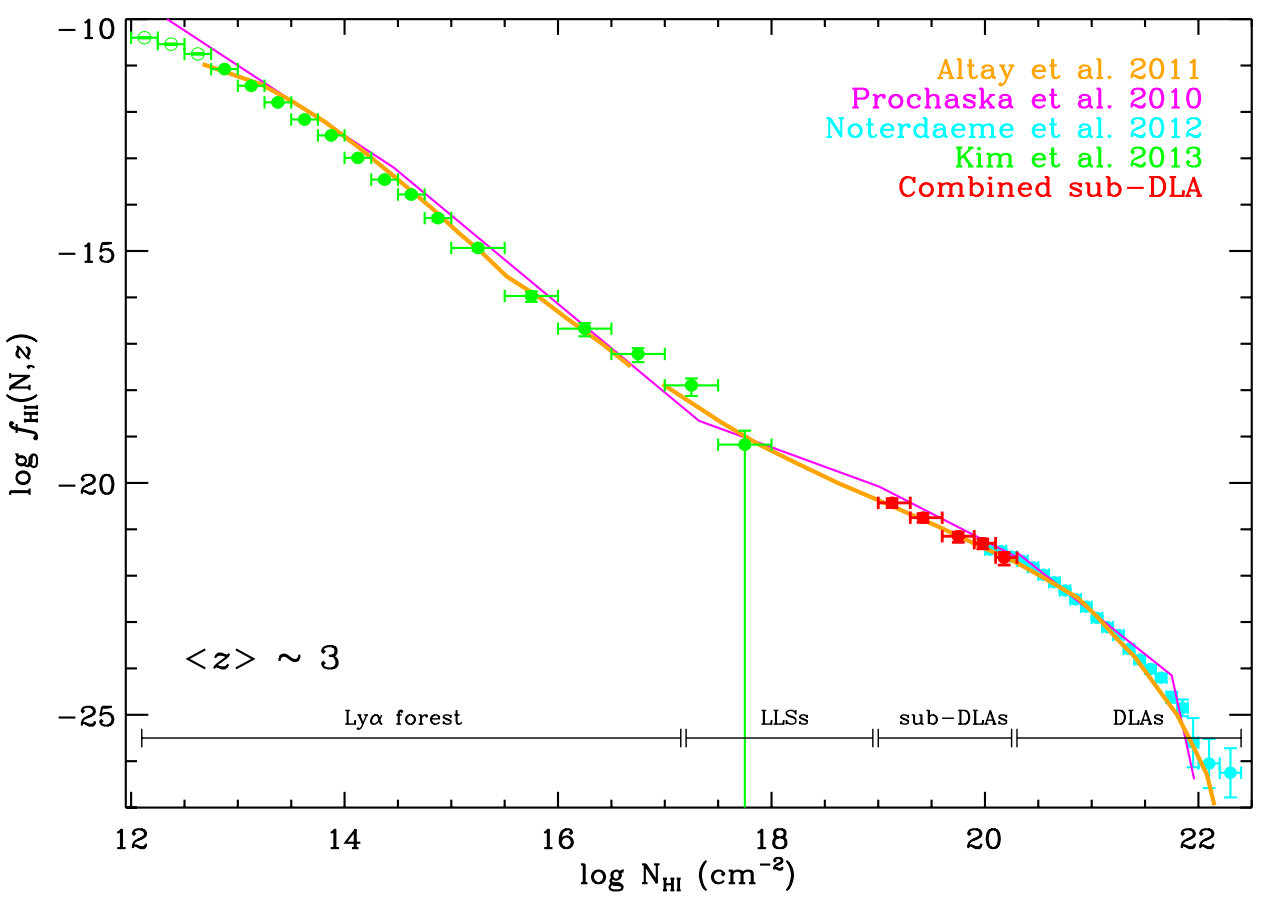

Fig. 8. Differential column density distribution $\left(f_{\mathrm{HI}}(N, z)\right)$ plotted against $\log N_{\mathrm{HI}}$. The red data points indicate $f_{\mathrm{HI}}(N, z)$ for sub-DLAs from the combined sub-DLA sample. The green filled data points represent $f_{\mathrm{HI}}(N, z)$ for Ly $\alpha$ forest from Kim et al. (2013). The green open circles are column densities from Kim et al. (2013) affected by incompleteness. The cyan data points represent the results from Noterdaeme et al. (2012). The solid magenta line is the estimate of $f_{N_{\mathrm{HI}}}(N, z)$ at $z \sim 3.7$ using a series of six power-laws by Prochaska et al. (2010). The solid orange line is hydrodynamic/analytical model prediction at $z \sim 3$ by Altay et al. (2011). and Péroux et al. (2003b). In the sub-DLA regime, the analysis comes from the combined sub-DLA sample. In the Ly $\alpha$ forest and LLS regimes, the results come from Kim et al. (2013), who used UVES observations of 18 quasar spectra at $1.9<z<3.2$. As can be seen in Fig. 8, there is a turnover in the distribution function at $\log N_{\mathrm{HI}}<12.7$ that is caused by the incompleteness of the sample at these column densities. The complete LLS regime remains uncategorized because the column densities are in the logarithmic part of the curve-of-growth.
In Fig. 8 a prediction from the hydrodynamical/analytical model of Altay et al. (2011), treating self-shielding and formation of molecular hydrogen in post-processing, is also plotted. Their low $N_{\mathrm{HI}}$ is obtained by fitting mock spectra with VPFIT and their high $N_{\mathrm{HI}}$ by projecting the simulation box onto a plane. A gap between low and high $N_{\mathrm{HI}}$ can also be clearly seen in Fig. 8. The Altay et al. (2011) model provides a reasonable representation of the data down to $\operatorname{Ly} \alpha$ forest range at $z \sim 3$ (see also van de Voort et al. 2012; Rahmati et al. 2013a,b). 
Table 7. Redshift distribution of the neutral gas mass density, $\Omega_{\mathrm{g}}$, in DLAs from Péroux et al. (2003b) and combined sub-DLA sample expressed as a function of the current critical density.

\begin{tabular}{|c|c|c|c|c|c|c|c|c|c|c|c|c|}
\hline \multirow{2}{*}{$\begin{array}{l}\log N_{\mathrm{HI}} \text { range } \\
z \text { range }\end{array}$} & \multicolumn{4}{|c|}{$\log N_{\mathrm{HI}}>20.3$} & \multirow[b]{2}{*}{$\begin{array}{c}\# \\
\text { DLAs } \\
\end{array}$} & \multirow[b]{2}{*}{$\langle z\rangle$} & \multirow[b]{2}{*}{$\mathrm{d} z$} & \multicolumn{3}{|c|}{$19.0<\log N_{\mathrm{HI}}<20.3$} & \multirow{2}{*}{$\begin{array}{c}\text { Total } \\
\boldsymbol{\Omega}_{\mathrm{g}} \\
\times 10^{-3} \\
\end{array}$} & \multirow[b]{2}{*}{$\begin{array}{c}\rho_{\mathrm{g}} \times 10^{8} \\
M_{\odot} \mathrm{Mpc}^{-3}\end{array}$} \\
\hline & $\langle z\rangle$ & $\mathrm{d} z$ & $\mathrm{~d} X$ & $\begin{array}{c}\Omega_{\mathrm{DLA}} \\
\times 10^{-3} \\
\end{array}$ & & & & $\mathrm{~d} X$ & $\begin{array}{c}\Omega_{\text {sub-DLA }} \\
\times 10^{-3}\end{array}$ & $\begin{array}{c}\# \\
\text { sub-DLAs }\end{array}$ & & \\
\hline 1.51 & 1.84 & 95.6 & 279.4 & $83 \pm$ & 21 & 1.80 & 29.9 & 87.3 & 0.0 & 4 & $.90 \pm 0.20$ & $1.23 \pm 0.33$ \\
\hline $2.00-2.50$ & 2.27 & 125.1 & 396.6 & $0.90 \pm$ & 26 & 2.26 & 49.2 & 156.8 & 0. & 1 & 29 & 1. \\
\hline $2.50-3.00$ & 2.73 & 87.2 & 301.0 & $0.96 \pm 0.28$ & 18 & 2.76 & 47.1 & 162.8 & $0.17 \pm 0.04$ & 24 & $1.13 \pm 0.28$ & 1.54 \\
\hline $3.00-3.50$ & 3.25 & 64.8 & 239.6 & $1.00 \pm 0.28$ & 18 & 3.21 & 33.7 & 124.5 & $0.16 \pm 0.05$ & 23 & $1.16 \pm 0.29$ & 1.58 \\
\hline $3.50-4.00$ & 3.77 & 49.5 & 194.6 & $0.75 \pm 0.21$ & 19 & 3.72 & 23.3 & 91.7 & $0.18 \pm 0.05$ & 19 & $1.03 \pm 0.21$ & 1.40 \\
\hline $4.00-5.00$ & 4.20 & 23.2 & 95.6 & $0.76 \pm 0.25$ & 10 & 4.18 & 9.9 & 41.0 & $0.20 \pm 0.09$ & 8 & $0.96 \pm 0.25$ & $1.31 \pm 0.36$ \\
\hline
\end{tabular}

Notes. The total amount of H I gas from DLAs + sub-DLAs is given in boldface. In the last column, we provide the H I mass density for DLA + sub-DLA.

\subsection{Neutral gas mass density}

The cosmological mass density of neutral gas, $\Omega_{\mathrm{g}}$, observed in high-redshift quasar absorbers is expressed as a fraction of the current critical density (see Lanzetta et al. 1995):

$\Omega_{\mathrm{g}}(z)=\frac{\mu m_{\mathrm{H}} H_{0}}{c \rho_{\text {crit }}} \int_{N_{\min }}^{N_{\max }} N_{\mathrm{HI}} f_{\mathrm{HI}}(N, z) \mathrm{d} N$,

where $f_{\mathrm{HI}}(N, z)$ is the $N_{\mathrm{HI}}$ frequency distribution, $m_{\mathrm{H}}$ is the mass of the hydrogen atom, $\rho_{\text {cric }}$ is the current critical mass density given as $\rho_{\text {cric }}=3 H_{0}^{2} / 8 \pi G$, and $\mu=1.3$ is the mean molecular mass of the gas used to incorporate helium as well as hydrogen in the estimate of the neutral gas density. No correction was applied for missing sub-DLAs because of dust (Pontzen \& Pettini 2009). Setting $N_{\min }=1 \times 10^{19}$ and $N_{\max }=2 \times 10^{20}$ gives the mass density of neutral gas for sub-DLAs, $\Omega_{\text {sub-DLA, and set- }}$ ting $N_{\min }=2 \times 10^{20}$ and $N_{\max }=\infty$ gives the mass density of neutral gas for DLAs, $\Omega_{\text {DLA }}$. It is worth mentioning that $f_{\mathrm{HI}}(N, z)$ steepens in the Ly $\alpha$ forest regime (see Fig. 8). A significant fraction of gas is photoionized at these column densities, therefore, the Ly $\alpha$ forest contributes less than one percent to $\Omega_{\mathrm{g}}$ (Kim et al. 2013). In the discrete limit, $\Omega_{\mathrm{g}}$ is given by

$\Omega_{\mathrm{g}}=\frac{\mu m_{\mathrm{H}} H_{0}}{c \rho_{\text {crit }}} \frac{\sum N_{\mathrm{HI}}}{\Delta X}$,

where the sum is calculated for sub-DLAs and DLAs along lines of sight with a total absorption path-length $\Delta X$. In Table 7 we provide total gas mass density for this work, including contribution from DLAs (Péroux et al. 2003b) and sub-DLAs (combined sub-DLA). The total gas mass density, including $\mathrm{H}$ I gas in absorbers up to the canonical sub-DLA definition, is plotted in Fig. 9. The binning for our data was selected with an increment of one half in redshift except for $4<z<5$ because of low number statistics. Vertical error bars correspond to $1 \sigma$ uncertainties and the horizontal error bars indicate bin sizes. It is a difficult task to measure $\Omega_{\mathrm{g}}$ at intermediate and low redshifts. For comparison, the H I mass density measured from radio observations of local galaxies using 21-cm emission is plotted in Fig. 9 (triangles at $z=0$ ). These observations come from the Westerbork H I survey of spiral and irregular galaxies (WHISP) project (Zwaan et al. 2005a), the $40 \%$ of the Arecibo legacy fast ALFA (ALFALFA) aurvey (Martin et al. 2010), and M31, M33, and the LMC (Braun 2012). The measurement at $z=0.24$ comes from the radio observations of star-forming galaxies (Lah et al. 2007). Rao et al. (2006) searched for the Ly $\alpha$ absorption associated to Mg II systems to measure $\Omega_{\mathrm{g}}$ at $0.1<z<1.7$. Their results and the measurements from the radio survey of Lah et al. (2007) are consistent with high-redshift findings, indicating that H I mass density

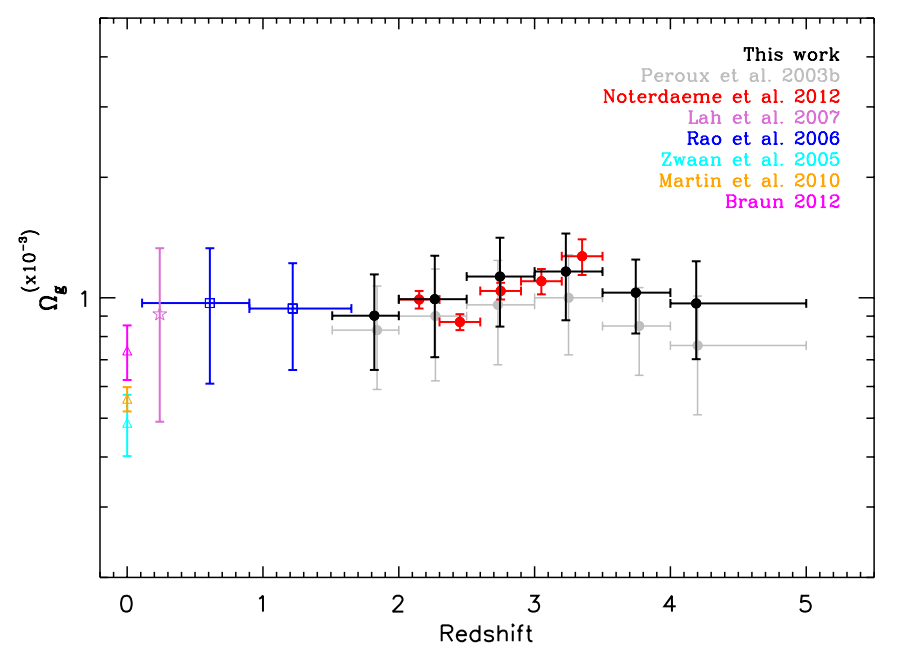

Fig. 9. Redshift evolution of the neutral gas mass density, $\Omega_{\mathrm{g}}$. The black bins correspond to $\Omega_{\mathrm{g}}$ measured from DLAs (Péroux et al. 2003b) plus sub-DLAs (combined sub-DLA) contribution. The gray bins show the DLA contribution to the $\Omega_{\mathrm{g}}$ from Péroux et al. (2003b) in the same redshift bins. The triangles at $z=0$ represent the $\Omega_{\mathrm{g}}$ measured in local galaxies by Zwaan et al. (2005a, cyan), Martin et al. (2010, orange), and Braun (2012, magenta), respectively. The open purple star at $z=0.24$ illustrates the H I content measured from the star-forming galaxies by Lah et al. (2007). The blue squares at $z<2$ represent the H I measured from $\mathrm{Mg}$ II selected DLAs by Rao et al. (2006). The red bins correspond to the $\Omega_{\mathrm{g}}$ measured from SDSS by Noterdaeme et al. (2012).

is roughly constant from $z \sim 0.1-5.0$. At $z=0$ the $21-\mathrm{cm}$ radio surveys (Zwaan et al. 2005a; Martin et al. 2010; Braun 2012) indicate a decrease in $\Omega_{\mathrm{g}}$ by at most a factor of 2 and a constant compatible with uncertainties. The observations of $21-\mathrm{cm}$ radio emission of neutral hydrogen are limited to low redshift (Zwaan et al. 2005a; Lah et al. 2007). More distant galaxies are generally too faint for an individual detection at $21 \mathrm{~cm}$. Recently, Chang et al. (2010) used $21-\mathrm{cm}$ maps to compute $\Omega_{\mathrm{g}}$ up to $z=0.8$.

The baryons comprise a small fraction of the critical matterenergy density with $\Omega_{\mathrm{b}}=0.0455 \pm 0.0028$ (Komatsu et al. 2011). Most of the baryons resides in the photoionized Ly $\alpha$ forest $(28 \pm 11 \%)$ and shocked-heated warm-hot intergalactic medium (WHIM) at $T \approx 10^{5-7} \mathrm{~K}(25 \pm 8 \%$; see Shull et al. 2012). A small fraction of baryons is locked up in stars in galaxies $(7 \pm 2 \%)$, groups, and clusters (Shull et al. 2012, and references therein). Current tracers of WHIM include O VI absorption lines, broad Ly $\alpha$ absorbers, and X-ray absorption lines. Another $5 \pm 3 \%$ may reside in condensed gas phase in the 
circumgalactic medium (CGM) within the virial radius of galaxies (Shull et al. 2012). The cold gas mass density that is the interest of the current study, contribute around $\lesssim 2 \%$ to the baryon fraction at $z=0$ in the $\mathrm{H} \mathrm{I}, \mathrm{He} \mathrm{I}$ and $\mathrm{H}_{2}$ phases . From the study of the multiphase mass densities in the present-day Universe, Shull et al. (2012) found that one third of the baryons are still missing. The $\Omega_{\mathrm{g}}$ at $z=0.1-5$ is $\sim 6$ and $\sim 12$ times smaller than the current $\Omega_{*+\mathrm{CGM}}$ and $\Omega_{\mathrm{WHIM}} \Omega_{\mathrm{g}}$ at $z=0.1-5.0$ is 45 times smaller than $\Omega_{\mathrm{b}}$.

\subsubsection{The contribution of sub-DLAs}

Péroux et al. (2003a) first derived the contribution of sub-DLAs to the $\Omega_{\mathrm{g}}$ as a function of the $N_{\mathrm{HI}}$ column density. They showed that by including sub-DLAs, most of the neutral gas mass in the Universe is accounted for. Noterdaeme et al. (2009) found that sub-DLAs contribute about $20 \%$ (from $\Gamma$-function extrapolation) to $30 \%$ (from double power-law extrapolation) to the total neutral gas mass. While the high column density systems dominate the contribution to $\Omega_{\mathrm{g}}$, the relative contribution of each class of absorbers may evolve. In addition, a nearly complete census of neutral gas mass in the Universe can be obtained by including the contribution of sub-DLAs to $\Omega_{\mathrm{g}}$. From a direct measurement, we find here that sub-DLAs contribute between $8-20 \%$ to $\Omega_{\mathrm{g}}$ with increasing contribution at high redshift. The sub-DLA contribution increases notably at $z \geq 3$, indicating that we are observing the assembly of high column density systems from low column density units. Our findings agree with those of Péroux et al. (2005) and Noterdaeme et al. (2009, 2012), suggesting no significant evolution of $\Omega_{\mathrm{g}}$ over redshift.

\subsubsection{Interpretation of the non evolution of $\Omega_{\mathrm{g}}$}

Previously, an evolution of $\Omega_{\mathrm{g}}$ was expected over cosmic time due to the consumption of neutral gas by star-formation activity. The evolution is also expected because energy is released during the hierarchical build-up of systems from subsystems (e.g., Ledoux et al. 1998) and/or ejection of gas through galactic winds from the central parts of massive halos into the IGM (Fall \& Pei 1993). Prochaska et al. (2005) and Prochaska \& Wolfe (2009) observed a significant decrease in H I mass between $z=2-4$ from the SDSS surveys. They attributed it to the consumption of neutral gas by star formation activity and/or ejection of gas into the IGM through galactic winds. Zwaan et al. (2008) and Tescari et al. (2009) debated the importance of galactic winds for the evolution of $\Omega_{\mathrm{g}}$.

In recent years, new observations have considerably changed the genetal picture of the observable baryons in the Universe (e.g, Cole et al. 2001; Fukugita \& Peebles 2004; Danforth \& Shull 2008; Shull et al. 2012; Noterdaeme et al. 2012). The H I clouds form molecules, and the molecular clouds then cool, fragment, and initiate star formation in galaxies. Therefore DLAs provide the reservoir of neutral gas and serve as a barometer of recent starformation activity. The neutral gas mass density $\rho_{\mathrm{g}}=\Omega_{\mathrm{g}} \rho_{\text {crit }}\left(\right.$ where $\left.\rho_{\text {crit }}=2.78 \times 10^{11} h^{2} M_{\odot} \mathrm{Mpc}^{-3}\right)$ has been measured over most of the age of the Universe (lookback time of about $12 \mathrm{Gyr}$ ). The space density of $\mathrm{H} \mathrm{I}$ appears to evolve surprisingly little from $z=0.1-5.0$. In contrast, the space density of the starformation rate (SFR) in galaxies steadily increases toward $z \simeq 2$ by almost an order of magnitude (e.g, Cucciati et al. 2012). Given the SFR density, it is expected that H I plus $\mathrm{H}_{2}$ gas at high-redshift would be exhausted on timescales of a few Gyr. Figure 10 indicates that there is no evolution of $\rho_{\mathrm{HI}}$

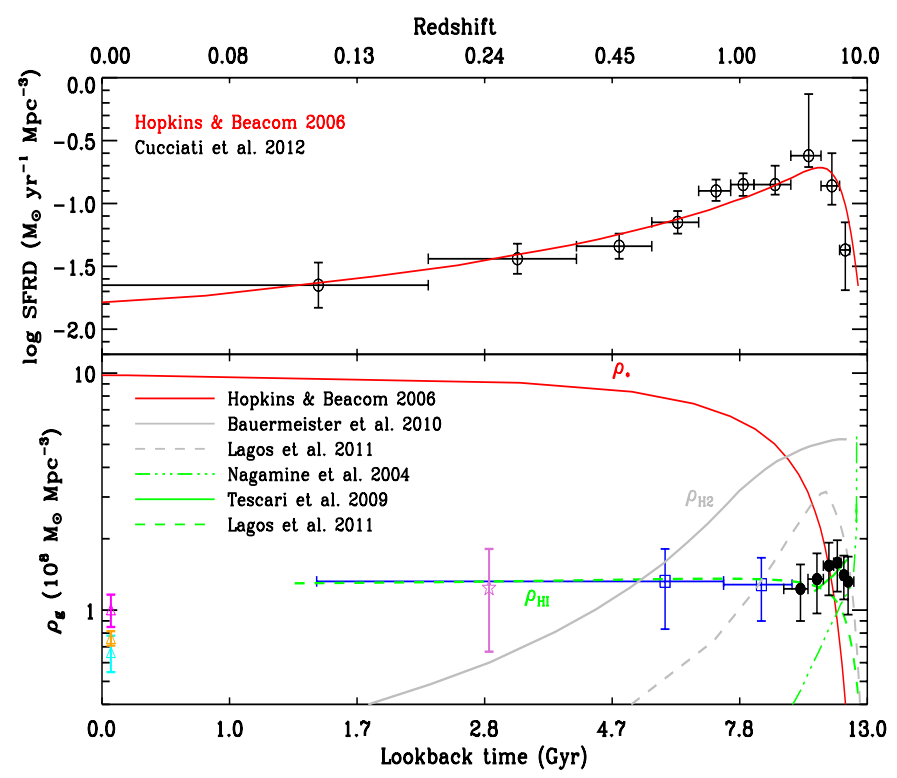

Fig. 10. Top panel: total dust-corrected UV-derived SFR density as a function of lookback time from the visible multi object-spectrograph (VIMOS)-VLT deep survey (VVDS) Deep + Ultra-Deep sample from Cucciati et al. (2012) is illustrated as black open circles. The red curve corresponds to the best-fitting parametric form to the SFR density from Hopkins \& Beacom (2006). Bottom panel: the data points have the same meaning as in Fig. 9. The green curves represent the prediction of $\rho_{\mathrm{HI}}$ from Nagamine et al. (2004, green triple dot-dashed line), Tescari et al. (2009, green solid line), and Lagos et al. (2011, green dashed line). The gray curves correspond to the prediction of $\rho_{\mathrm{H}_{2}}$ from Bauermeister et al. (2010, solid gray line) and Lagos et al. (2011, gray dashed line). The solid red line illustrates the evolution of the stellar mass density build-up inferred from the star formation history by Hopkins \& Beacom (2006).

over a lookback time of 1.3-12.3 Gyr. This suggests that SFR, ionization of neutral gas, and/or the formation of molecular hydrogen alone cannot explain the non-evolution of the H I space density.

Several surveys have been conducted to study inflows and outflows in distant galaxies via UV absorption lines. Outflows are ubiquitous in almost all distant star-forming galaxies (Weiner et al. 2009; Rubin et al. 2010; Steidel et al. 2010; Kacprzak et al. 2011; Bordoloi et al. 2011) and their rates are similar to or higher than the SFR (e.g., Kornei et al. 2012). Recently, cool gas inflows have been detected in a few galaxies, although with an inflow rate lower than the SFR (Sato et al. 2009; Giavalisco et al. 2011; Rubin et al. 2012; Martin et al. 2012). Because the outflow rates are generally found to be higher than the SFR, a constant HI space density as we observe seems to indicate a higher accretion on average than observed in the quoted observations.

Introducing a continuous replenishment of gas the account for an appropriate evolution of outflows and gas replenishment rates, i.e., accretion of gas from filaments and streams to the centers of cold dark matter halos, can possibly help in resolving the puzzle of the non-evolution of the space density of H I (Hopkins et al. 2008). Hydrodynamical simulation indicate that H I gas might be replenished by accretion of matter from the IGM and/or recombination of ionized gas in the walls of supershells (Kereš et al. 2005; Birnboim et al. 2007; Erb 2008). 


\section{Conclusions}

We here presented a statistical sample of 122 quasars $(1.5<$ $\left.z_{\mathrm{em}}<5.0\right)$ observed with the VLT/UVES. The statistical properties of 27 sub-DLAs along the lines of sight of these quasars were analyzed in combination with the sub-DLA studies of Péroux et al. (2003a, 2005) and O'Meara et al. (2007). Our main findings are summarized as follows:

- The combined sub-DLA sample allows one to determine the redshift evolution of the number density and line density. Comparing these with DLAs and LLSs results from the literature, we found that all three classes of absorbers are evolving in the redshift range $1<z<5$ in the same way.

- The shape of the column density distribution function down to $\log N_{\mathrm{HI}}=19.0$ was determined. The redshift evolution of $f_{\mathrm{HI}}(N, z)$ was also presented for two redshift bins $1.5<z<$ 3.1 and $3.1<z<5.0$. The shape of $f_{\mathrm{HI}}(N, z)$ at high-redshift in the sub-DLA range is steeper, indicating that there are more sub-DLAs at high-redshift than at low-redshift. This evolution suggests that sub-DLAs may merge and/or ionize over cosmic time.

- We furthermore used $f_{\mathrm{HI}}(N, z)$ to determine the total H I gas mass in the Universe at $1.5<z<5.0$. The complete sample shows that sub-DLAs are significant at all redshifts and their contribution to $\Omega_{\mathrm{g}}$ increases from 8-20\% with an increasing fraction at higher redshift.

- From $0.1<z<5.0$ no-evolution of $\Omega_{\mathrm{g}}$ was found. In contrast, star formation peaks at $z \sim 2.1$. This indicates that star formation aline cannot explain the non-evolution of $\Omega_{\mathrm{g}}$. This may suggest that replenishment of gas through accretion of matter from filaments and/or recombination of ionized gas in the walls of supershells may help in sustaining $\Omega_{\mathrm{g}}$ as a constant over cosmic time (Hopkins et al. 2008).

Acknowledgements. This work has been funded within the BINGO! ("history of Baryons: INtergalactic medium/Galaxies cO-evolution") project by the Agence Nationale de la Recherche (ANR) under the allocation ANR-08-BLAN-0316-01. We are thankful to the anonymous referee for his/her constructive comments. We would like to thank the ESO staff for making the UVES Advanced Data Products available to the community.

\section{References}

Altay, G., Theuns, T., Schaye, J., Crighton, N. H. M., \& Dalla Vecchia, C. 2011, ApJ, 737, L37

Bahcall, J. N., \& Peebles, P. J. E. 1969, ApJ, 156, L7

Bauermeister, A., Blitz, L., \& Ma, C.-P. 2010, ApJ, 717, 323

Birnboim, Y., Dekel, A., \& Neistein, E. 2007, MNRAS, 380, 339

Bordoloi, R., Lilly, S. J., Knobel, C., et al. 2011, ApJ, 743, 10

Braun, R. 2012, ApJ, 749, 87

Carswell, R. F., Webb, J. K., Lanzetta, K. M., et al. 1996, MNRAS, 278, 506

Chang, T.-C., Pen, U.-L., Bandura, K., \& Peterson, J. B. 2010, Nature, 466, 463

Cole, S., Norberg, P., Baugh, C. M., et al. 2001, MNRAS, 326, 255

Coppolani, F., Petitjean, P., Stoehr, F., et al. 2006, MNRAS, 370, 1804

Cucciati, O., Tresse, L., Ilbert, O., et al. 2012, A\&A, 539, A31

Danforth, C. W., \& Shull, J. M. 2008, ApJ, 679, 194

Dekker, H., D’Odorico, S., Kaufer, A., Delabre, B., \& Kotzlowski, H. 2000, in SPIE Conf. Ser. 4008, eds. M. Iye, \& A. F. Moorwood, 534

Dessauges-Zavadsky, M., Péroux, C., Kim, T.-S., D’Odorico, S., \& McMahon, R. G. 2003, MNRAS, 345, 447

Dessauges-Zavadsky, M., Ellison, S. L., \& Murphy, M. T. 2009, MNRAS, 396, L61

D’Odorico, V., Petitjean, P., \& Cristiani, S. 2002, A\&A, 390, 13

Ellison, S. L., \& Lopez, S. 2009, MNRAS, 397, 467

Ellison, S. L., Pettini, M., Steidel, C. C., \& Shapley, A. E. 2001a, ApJ, 549, 770

Ellison, S. L., Yan, L., Hook, I. M., et al. 2001b, A\&A, 379, 393

Erb, D. K. 2008, ApJ, 674, 151
Erkal, D., Gnedin, N. Y., \& Kravtsov, A. V. 2012, ApJ, 761, 54

Fall, S. M., \& Pei, Y. C. 1993, ApJ, 402, 479

Fontana, A., \& Ballester, P. 1995, The Messenger, 80, 37

Fox, A. J., Prochaska, J. X., Ledoux, C., et al. 2009, A\&A, 503, 731

Fox, A. J., Ledoux, C., Petitjean, P., Srianand, R., \& Guimarães, R. 2011, A\&A, 534, A82

Fukugita, M., \& Peebles, P. J. E. 2004, ApJ, 616, 643

Giavalisco, M., Vanzella, E., Salimbeni, S., et al. 2011, ApJ, 743, 95

Guimarães, R., Petitjean, P., de Carvalho, R. R., et al. 2009, A\&A, 508, 133

Herbert-Fort, S., Prochaska, J. X., Dessauges-Zavadsky, M., et al. 2006, PASP, 118,1077

Hopkins, A. M., \& Beacom, J. F. 2006, ApJ, 651, 142

Hopkins, A. M., McClure-Griffiths, N. M., \& Gaensler, B. M. 2008, ApJ, 682, L13

Ivanchik, A. V., Petitjean, P., Balashev, S. A., et al. 2010, MNRAS, 404, 1583

Kacprzak, G. G., Churchill, C. W., Evans, J. L., Murphy, M. T., \& Steidel, C. C. 2011, MNRAS, 416, 3118

Kanekar, N., Smette, A., Briggs, F. H., \& Chengalur, J. N. 2009, ApJ, 705, L40

Kaplan, K. F., Prochaska, J. X., Herbert-Fort, S., Ellison, S. L., \& Dessauges-Zavadsky, M. 2010, PASP, 122, 619

Katz, N., Weinberg, D. H., Hernquist, L., \& Miralda-Escude, J. 1996, ApJ, 457, L57

Kereš, D., Katz, N., Weinberg, D. H., \& Davé, R. 2005, MNRAS, 363, 2

Kim, T.-S., Partl, A. M., Carswell, R. F., \& Müller, V. 2013, A\&A, 552, A77

Klypin, A., Borgani, S., Holtzman, J., \& Primack, J. 1995, ApJ, 444, 1

Komatsu, E., Smith, K. M., Dunkley, J., et al. 2011, ApJS, 192, 18

Kornei, K. A., Shapley, A. E., Martin, C. L., et al. 2012, ApJ, 758, 135

Krumholz, M. R., Ellison, S. L., Prochaska, J. X., \& Tumlinson, J. 2009, ApJ, 701, L12

Lagos, C. D. P., Baugh, C. M., Lacey, C. G., et al. 2011, MNRAS, 418, 1649

Lah, P., Chengalur, J. N., Briggs, F. H., et al. 2007, MNRAS, 376, 1357

Lanzetta, K. M., Wolfe, A. M., \& Turnshek, D. A. 1995, ApJ, 440, 435

Le Brun, V., Bergeron, J., Boisse, P., \& Deharveng, J. M. 1997, A\&A, 321, 733

Ledoux, C., Petitjean, P., Bergeron, J., Wampler, E. J., \& Srianand, R. 1998, A\&A, 337, 51

Ledoux, C., Bergeron, J., \& Petitjean, P. 2002, A\&A, 385, 802

Ledoux, C., Petitjean, P., \& Srianand, R. 2003, MNRAS, 346, 209

Ledoux, C., Petitjean, P., Fynbo, J. P. U., Møller, P., \& Srianand, R. 2006, A\&A, 457, 71

Lopez, S., Reimers, D., Rauch, M., Sargent, W. L. W., \& Smette, A. 1999, ApJ, 513,598

Lopez, S., Reimers, D., Gregg, M. D., et al. 2005, ApJ, 626, 767

Ma, C.-P., \& Bertschinger, E. 1994, ApJ, 434, L5

Martin, A. M., Papastergis, E., Giovanelli, R., et al. 2010, ApJ, 723, 1359

Martin, C. L., Shapley, A. E., Coil, A. L., et al. 2012, ApJ, 760, 127

McQuinn, M., Oh, S. P., \& Faucher-Giguère, C.-A. 2011, ApJ, 743, 82

Nagamine, K., Springel, V., \& Hernquist, L. 2004, MNRAS, 348, 421

Noterdaeme, P., Ledoux, C., Petitjean, P., et al. 2007, A\&A, 474, 393

Noterdaeme, P., Ledoux, C., Petitjean, P., \& Srianand, R. 2008a, A\&A, 481, 327

Noterdaeme, P., Petitjean, P., Ledoux, C., Srianand, R., \& Ivanchik, A. 2008b, A\&A, 491, 397

Noterdaeme, P., Petitjean, P., Ledoux, C., \& Srianand, R. 2009, A\&A, 505, 1087

Noterdaeme, P., Petitjean, P., Carithers, W. C., et al. 2012, A\&A, 547, L1

O'Meara, J. M., Prochaska, J. X., Burles, S., et al. 2007, ApJ, 656, 666

O’Meara, J. M., Prochaska, J. X., Worseck, G., Chen, H.-W., \& Madau, P. 2013, ApJ, 765, 137

Péroux, C., Storrie-Lombardi, L. J., McMahon, R. G., Irwin, M., \& Hook, I. M. 2001, AJ, 121, 1799

Péroux, C., Dessauges-Zavadsky, M., D’Odorico, S., Kim, T.-S., \& McMahon, R. G. 2003a, MNRAS, 345, 480

Péroux, C., McMahon, R. G., Storrie-Lombardi, L. J., \& Irwin, M. J. 2003b, MNRAS, 346, 1103

Péroux, C., Deharveng, J.-M., Le Brun, V., \& Cristiani, S. 2004, MNRAS, 352 , 1291

Péroux, C., Dessauges-Zavadsky, M., D’Odorico, S., Sun Kim, T., \& McMahon, R. G. 2005, MNRAS, 363, 479

Pontzen, A., \& Pettini, M. 2009, MNRAS, 393, 557

Pontzen, A., Governato, F., Pettini, M., et al. 2008, MNRAS, 390, 1349

Prochaska, J. X., \& Herbert-Fort, S. 2004, PASP, 116, 622

Prochaska, J. X., \& Wolfe, A. M. 2009, ApJ, 696, 1543

Prochaska, J. X., Herbert-Fort, S., \& Wolfe, A. M. 2005, ApJ, 635, 123

Prochaska, J. X., Wolfe, A. M., Howk, J. C., et al. 2007, ApJS, 171, 29

Prochaska, J. X., O’Meara, J. M., \& Worseck, G. 2010, ApJ, 718, 392

Quast, R., Reimers, D., \& Baade, R. 2008, A\&A, 477, 443

Rahmati, A., Pawlik, A. H., Raicevic, M., \& Schaye, J. 2013a, MNRAS, 430, 2427 
T. Zafar et al.: Cosmological evolution of the neutral gas mass density

Rahmati, A., Schaye, J., Pawlik, A. H., \& Raicevic, M. 2013b, MNRAS, 431, 2261

Rao, S. M., Turnshek, D. A., \& Nestor, D. B. 2006, ApJ, 636, 610

Ribaudo, J., Lehner, N., \& Howk, J. C. 2011, ApJ, 736, 42

Richter, P., Ledoux, C., Petitjean, P., \& Bergeron, J. 2005, A\&A, 440, 819

Rix, S. A., Pettini, M., Steidel, C. C., et al. 2007, ApJ, 670, 15

Rubin, K. H. R., Weiner, B. J., Koo, D. C., et al. 2010, ApJ, 719, 1503

Rubin, K. H. R., Prochaska, J. X., Koo, D. C., \& Phillips, A. C. 2012, ApJ, 747, L26

Sato, T., Martin, C. L., Noeske, K. G., Koo, D. C., \& Lotz, J. M. 2009, ApJ, 696, 214

Schaye, J. 2001, ApJ, 562, L95

Shull, J. M., Smith, B. D., \& Danforth, C. W. 2012, ApJ, 759, 23

Songaila, A., \& Cowie, L. L. 2010, ApJ, 721, 1448

Spergel, D. N., Verde, L., Peiris, H. V., et al. 2003, ApJS, 148, 175

Srianand, R., \& Petitjean, P. 1998, A\&A, 335, 33

Srianand, R., Gupta, N., \& Petitjean, P. 2007, MNRAS, 375, 584
Steidel, C. C., Erb, D. K., Shapley, A. E., et al. 2010, ApJ, 717, 289

Storrie-Lombardi, L. J., \& Wolfe, A. M. 2000, ApJ, 543, 552

Tescari, E., Viel, M., Tornatore, L., \& Borgani, S. 2009, MNRAS, 397, 411

Tytler, D. 1987, ApJ, 321, 49

van de Voort, F., Schaye, J., Altay, G., \& Theuns, T. 2012, MNRAS, 421, 2809

Viegas, S. M. 1995, MNRAS, 276, 268

Weiner, B. J., Coil, A. L., Prochaska, J. X., et al. 2009, ApJ, 692, 187

Wolfe, A. M., Lanzetta, K. M., Foltz, C. B., \& Chaffee, F. H. 1995, ApJ, 454, 698

Zafar, T., Popping, A., \& Péroux, C. 2013, A\&A, 556, A140 (Paper I)

Zwaan, M. A., Meyer, M. J., Staveley-Smith, L., \& Webster, R. L. 2005a, MNRAS, 359, L30

Zwaan, M. A., van der Hulst, J. M., Briggs, F. H., Verheijen, M. A. W., \& Ryan-Weber, E. V. 2005b, MNRAS, 364, 1467

Zwaan, M., Walter, F., Ryan-Weber, E., et al. 2008, AJ, 136, 2886 
Table 1. Complete log of 250 EUADP quasars of which 122 contribute to the statistical EUADP sample.

\begin{tabular}{|c|c|c|c|c|c|c|}
\hline Quasar & $z_{\mathrm{em}}$ & $z_{\min }-z_{\max }$ & $z_{\mathrm{LLS}}$ & $z_{\mathrm{abs}}$ & $\begin{array}{c}\log N_{\mathrm{HI}} \\
\mathrm{cm}^{-2} \\
\end{array}$ & Ref. \\
\hline LBQS 2359-0216B & 2.810 & $1.542-2.083$ & $\ldots$ & 2.0952 & $20.65 \pm 0.10$ & 1 \\
\hline & $\ldots$ & $2.161-2.772$ & $\ldots$ & 2.1542 & $20.30 \pm 0.10$ & 1 \\
\hline QSO J0003-2323 & 2.280 & $1.509-2.247$ & $\ldots$ & 2.1871 & $19.60 \pm 0.40$ & 2 \\
\hline QSO B0002-422 & 2.760 & $1.509-2.722$ & $\ldots$ & $\cdots$ & $\cdots$ & \\
\hline QSO J0006-6208 & 4.455 & $3.083-3.188$ & $\cdots$ & 2.970 & 20.70 & 3 \\
\hline$\ldots$ & $\ldots$ & $3.216-3.732$ & $\ldots$ & 3.2022 & $20.80 \pm 0.10$ & 4 \\
\hline$\ldots$ & $\ldots$ & $3.798-4.400$ & $\ldots$ & 3.776 & 21.00 & 3 \\
\hline$\ldots$ & $\ldots$ & & $\ldots$ & 4.1451 & $19.37 \pm 0.15$ & 4 \\
\hline $\begin{array}{l}\text { QSO J0008-0958 } \\
\ldots\end{array}$ & $\begin{array}{c}1.950 \\
\ldots\end{array}$ & $\begin{array}{l}1.715-1.754 \\
1.782-1.921\end{array}$ & $\begin{array}{l}\cdots \\
\cdots\end{array}$ & $\begin{array}{l}1.7682 \\
\ldots\end{array}$ & $\begin{array}{c}20.85 \pm 0.15 \\
\ldots\end{array}$ & 5 \\
\hline QSO J0008-2900 & 2.645 & $1.715-2.609$ & $\ldots$ & 2.2542 & $20.22 \pm 0.10$ & 6 \\
\hline QSO J0008-2901 & 2.607 & $1.715-2.571$ & $\cdots$ & 2.4911 & $19.94 \pm 0.11$ & 6 \\
\hline QSO B0008+006 & 2.309 & $2.093-2.276$ & $\ldots$ & $\ldots$ & . & \\
\hline LBQS 0009-0138 & 1.998 & $1.715-1.968$ & $\cdots$ & 1.3862 & $20.26 \pm 0.02$ & 7 \\
\hline $\begin{array}{l}\text { LBQS 0010-0012 } \\
\ldots\end{array}$ & $\begin{array}{c}2.145 \\
\ldots\end{array}$ & $\begin{array}{l}1.509-2.009 \\
2.041-2.114\end{array}$ & $\begin{array}{l}\ldots \\
\ldots\end{array}$ & $\begin{array}{l}2.0252 \\
\ldots\end{array}$ & $\begin{array}{c}20.95 \pm 0.10 \\
\ldots\end{array}$ & 1 \\
\hline $\begin{array}{l}\text { LBQS 0013-0029 } \\
\ldots\end{array}$ & $\begin{array}{c}2.087 \\
\ldots\end{array}$ & $\begin{array}{l}1.512-1.958 \\
1.988-2.056\end{array}$ & $\begin{array}{l}\cdots \\
\ldots\end{array}$ & $\begin{array}{l}1.968 \\
1.9732\end{array}$ & $\begin{array}{c}\lesssim 19.43 \\
20.83 \pm 0.05\end{array}$ & $\begin{array}{l}8 \\
1\end{array}$ \\
\hline LBQS 0018+0026 & 1.244 & 0 & $\ldots$ & 0.5201 & $19.54 \pm 0.03$ & 7 \\
\hline$\ldots$ & $\cdots$ & $\cdots$ & $\cdots$ & 0.9401 & $19.38 \pm 0.15$ & 7 \\
\hline $\begin{array}{l}\text { QSO B0027-1836 } \\
\ldots\end{array}$ & $\begin{array}{c}2.550 \\
\ldots\end{array}$ & $\begin{array}{l}1.553-1.862, \\
1.877-2.183\end{array}$ & $\begin{array}{l}2.400 \\
\ldots\end{array}$ & $\begin{array}{l}2.4022 \\
\ldots\end{array}$ & $\begin{array}{c}21.75 \pm 0.10 \\
\ldots\end{array}$ & 9 \\
\hline J004054.7-091526 & 4.976 & 4.534-4.729, & $\cdots$ & 4.5382 & $20.20 \pm 0.09$ & 6 \\
\hline$\ldots$ & $\ldots$ & $4.751-4.916$ & $\ldots$ & 4.7402 & $20.39 \pm 0.11$ & 6 \\
\hline QSO J0041-4936 & 3.240 & $1.706-2.236$ & $\ldots$ & 2.2482 & $20.46 \pm 0.13$ & 6 \\
\hline$\cdots$ & & $2.260-2.718$ & $\ldots$ & $\ldots$ & $\ldots$ & \\
\hline$\cdots$ & $\cdots$ & $2.800-3.198$ & $\cdots$ & $\cdots$ & $\cdots$ & \\
\hline QSO B0042-2450 & 0.807 & 0 & $\cdots$ & $\cdots$ & $\cdots$ & \\
\hline QSO B0039-407 & 2.478 & $1.710-2.443$ & $\cdots$ & $\cdots$ & $\cdots$ & \\
\hline $\begin{array}{l}\text { QSO B0039-3354 } \\
\ldots\end{array}$ & $\begin{array}{c}2.480 \\
\ldots\end{array}$ & $\begin{array}{l}1.710-2.212 \\
2.236-2.445\end{array}$ & $\begin{array}{l}\ldots \\
\ldots\end{array}$ & $\begin{array}{l}2.2242 \\
\ldots\end{array}$ & $\begin{array}{c}20.60 \pm 0.10 \\
\ldots\end{array}$ & 10 \\
\hline LBQS 0041-2638 & 3.053 & $2.090-3.012$ & $\ldots$ & $\cdots$ & $\cdots$ & \\
\hline LBQS 0041-2707 & 2.786 & $2.090-2.748$ & $\cdots$ & $\cdots$ & $\cdots$ & \\
\hline QSO B0042-2656 & 3.358 & $2.217-3.096$ & 3.288 & $\cdots$ & $\cdots$ & \\
\hline LBQS 0042-2930 & 2.388 & $1.706-1.798$ & $\cdots$ & 1.8092 & $20.40 \pm 0.10$ & 10 \\
\hline$\cdots$ & 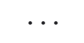 & 1.820-1.926, & $\cdots$ & 1.9362 & $20.50 \pm 0.10$ & 10 \\
\hline$\cdots$ & $\cdots$ & $1.946-2.354$ & $\cdots$ & $\cdots$ & $\cdots$ & \\
\hline LBC & 2.898 & $2.090-2.859$ & $\ldots$ & $\cdots$ & $\ldots$ & \\
\hline J004612.2-293110 & 1.675 & $1.550-1.648$ & $\ldots$ & $\cdots$ & $\cdots$ & \\
\hline
\end{tabular}

Notes. The columns provide the quasar names, emission redshifts, $z_{\min }$ (either LLS or S/N ratio threshold of $E W_{\text {rest }}=2.5 \AA$ ) and $z_{\max }$ (3000 $\mathrm{km} \mathrm{s}^{-1}$ blueward of the Ly $\alpha$ emission line), LLS redshifts, DLA/sub-DLA redshifts, H I column densities of DLAs/sub-DLAs and references for absorbers. The redshift path, $z_{\text {abs }}$ and $N_{\mathrm{HI}}$ of the absorbers included in our statistical sample are shown in boldface. For the redshift path surveyed, gaps in non-overlapping settings and known DLAs/subDLAs were excluded.

References. (1) Ledoux et al. (2006); (2) Richter et al. (2005); (3) Péroux et al. (2001); (4) Péroux et al. (2005); (5) Kaplan et al. (2010); (6) Paper I; (7) Dessauges-Zavadsky et al. (2009); (8) Ledoux et al. (2003); (9) Noterdaeme et al. (2007); (10) Fox et al. (2009); (11) Rao et al. (2006); (12) Ellison \& Lopez (2009); (13) Prochaska \& Herbert-Fort (2004); (14) Ellison et al. (2001a); (15) Péroux et al. (2003b); (16) Srianand et al. (2007); (17) D’Odorico et al. (2002); (18) Kanekar et al. (2009); (19) Noterdaeme et al. (2009); (20) Noterdaeme et al. (2008a); (21) Ellison et al. (2001b); (22) Carswell et al. (1996); (23) Péroux et al. (2004); (24) Lopez et al. (2005); (25) Quast et al. (2008); (26) Prochaska et al. (2007); (27) Srianand \& Petitjean (1998); (28) Fox et al. (2011); (29) Guimarães et al. (2009); (30) Lopez et al. (1999); (31) Ledoux et al. (2002); (32) Le Brun et al. (1997); (33) Dessauges-Zavadsky et al. (2003); (34) Ivanchik et al. (2010); (35) Herbert-Fort et al. (2006); (36) Noterdaeme et al. (2008b); (37) O'Meara et al. (2007); (38) Rix et al. (2007).
Table 1. continued.

\begin{tabular}{|c|c|c|c|c|c|c|}
\hline Quasar & $z_{\mathrm{em}}$ & $z_{\min }-z_{\max }$ & $z_{\mathrm{LLS}}$ & $z_{\mathrm{abs}}$ & $\begin{array}{c}\log N_{\mathrm{HI}} \\
\mathrm{cm}^{-2}\end{array}$ & Re \\
\hline 66 & 1.242 & 0 & $\ldots$ & $\ldots$ & .. & \\
\hline 0 & 36 & ( & $\ldots$ & $\ldots$ & $\ldots$ & \\
\hline 6 & 1.410 & 0 & $\ldots$ & $\ldots$ & .. & \\
\hline 8 & 1.969 & $1.706-1.939$ & $\ldots$ & $\ldots$ & .. & \\
\hline 5 & 2.082 & 1.710 & $\ldots$ & $\cdots$ & .. & \\
\hline 8 & 2.249 & $1.710-2$. & $\ldots$ & $\ldots$ & $\ldots$ & \\
\hline 5 & 1.528 & 0 & $\ldots$ & $\ldots$ & $\ldots$ & \\
\hline 05 & 0.624 & 0 & $\ldots$ & $\ldots$ & .. & \\
\hline 6 & 3.662 & $1.533-3.615$ & 2.377 & $\ldots$ & $\cdots$ & \\
\hline 92 & 3.093 & 1.77 & 2.695 & 2.67 & $10 \pm$ & 1 \\
\hline . & $\ldots 2$ & $2.105-2.656$ & $\ldots$ & $\ldots$ & $\ldots$ & \\
\hline . & $\ldots$ & 2.6 & $\cdots$ & $\cdots$ & $\cdots$ & \\
\hline & 1.595 & & $\cdots$ & $\ldots$ & $\cdots$ & \\
\hline & 2.686 & & $\ldots$ & 2.309 & $35=$ & 3 \\
\hline QSO J & 3.037 & & 2.941 & 2.3 & 88 & 310 \\
\hline & & & $\cdots$ & 2.926 & 10 & 10 \\
\hline & 2.220 & & $\cdots$ & $\ldots$ & $\cdots$ & \\
\hline & 3. & & $\cdots$ & $\ldots$ & $\cdots$ & \\
\hline & 2.4 & & $\ldots$ & $\ldots$ & $\ldots$ & \\
\hline QSO $\mathrm{F}$ & 2.985 & $2.930-2$ & $\ldots$ & 2.41 & 8 & 1 \\
\hline & & & $\cdots$ & 2.7 & $30=$ & 1 \\
\hline 1 & 1.609 & 0 & $\cdots$ & $\cdots$ & $\cdots$ & \\
\hline & 1.550 & 0 & $\cdots$ & 1.409 & $.08 \pm$ & 911 \\
\hline QSC & 3.834 & $2.426-3$ & $\cdots$ & 2.9 & $8 \pm$ & 4 \\
\hline & $\cdots$ & & .. & 3.078 & $21 \pm$ & 4 \\
\hline & 2.19 & 1 & $\cdots$ & & $\ldots$ & \\
\hline QS & 2.278 & & $\cdots$ & 1.7 & 07 & 712 \\
\hline & & & & & & \\
\hline & 1.900 & 1. & $\cdots$ & 1.857 & $20.21 \pm 0.09$ & \\
\hline & 3.023 & & $\cdots$ & $\cdots$ & $\cdots$ & \\
\hline QSC & 4.154 & & 4.14 & 3.139 & $1 \pm$ & 4 \\
\hline$\cdots$ & $\cdots$ & 3.35 & & & & 54 \\
\hline$\cdots$ & $\cdots$ & 3.7 & $\cdots$ & 3.7 & 10 & 4 \\
\hline$\cdots$ & $\cdots$ & $3.786-4.102$ & & 3.9 & 15 & 5 \\
\hline$\cdots$ & $\cdots$ & 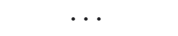 & 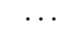 & 3.999 & 15 & 54 \\
\hline 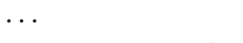 & & $\ldots$ & $\cdots$ & 4.0 & 15 & 54 \\
\hline & 1.522 & 0 & .. & & $1003+$ & 311 \\
\hline & 3.970 & $2.498-3$. & 3.662 & & 10 & \\
\hline & & & & & & 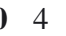 \\
\hline & 13 & & . & & & \\
\hline & 3.016 & 1 & - & 2.677 & $20.70 \pm 0.15$ & 5.13 \\
\hline & 1807 & $1.509-1.779$ & $\ldots$ & & & \\
\hline $2 \sqrt{3}$ & .831 & & & $0.1 / 1$ & $.70 \pm 0.09$ & $\Rightarrow 11$ \\
\hline
\end{tabular}
QSO J0153-4311 $2.789 \begin{array}{lllll}1.588-2.751 & 2.451 & \ldots\end{array}$

$\begin{array}{lllllll}\text { QSO J0157-0048 } & 1.545 & 0 & \cdots & 1.416 & 19.90 \pm 0.07 & 7\end{array}$ QSO B0201+113 3.610 2.309-2.661, 3.400 3.385 21.26 \pm 0.0814 $\begin{array}{lcccccc}\cdots & \cdots & \mathbf{2 . 7 0 8}-\mathbf{3 . 0 9 7} & \cdots & \cdots & \cdots & \\ \text { QSO J0209+0517 } & 4.174 & \mathbf{2 . 7 6 7}-\mathbf{3 . 6 5 8}, & \cdots & 3.666 & 20.47 \pm 0.10 & 4\end{array}$

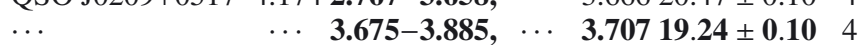
$\begin{array}{lllllll}\ldots & \ldots & \mathbf{3 . 8 7 2}-\mathbf{4 . 1 2 2} & \cdots & 3.863 & 20.43 \pm 0.15 & 4\end{array}$ $\begin{array}{llllll}\mathrm{J} 021741.8-370100 & 2.910 & \mathbf{1 . 7 1 5}-\mathbf{2 . 4 1 7}, \cdots & 2.429 & 20.62 \pm 0.08 \quad 6\end{array}$ $\begin{array}{lllllll}\ldots & \cdots & \mathbf{2 . 4 4 1}-\mathbf{2 . 5 0 3}, & \cdots & 2.51420 .46 \pm 0.09 & 6\end{array}$ $\begin{array}{lcccccc}\cdots & \cdots & \mathbf{2 . 5 2 5}-\mathbf{2 . 7 1 8} & \cdots & \cdots & \cdots & \\ \text { QSO J0217+0144 } & 1.715 & 0 & \cdots & 1.345 & 19.89 \pm 0.09 & 11\end{array}$

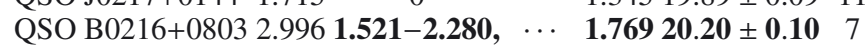

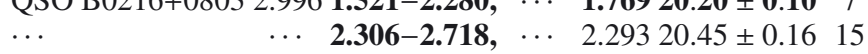
… $\quad \ldots \quad \mathbf{2 . 9 3 0 - 2 . 9 5 6}$ QSO B0227-369 2.115 1.715-2.084 QSO B0237-2322 $2.225 \quad 1.509-2.193 \quad \cdots \quad 1.36519 .30 \pm 0.30 \quad 16$ $\begin{array}{lllllll} & \ldots & \ldots & \ldots & 1.672 & 19.65 \pm 0.10 & 10\end{array}$

Q 2.071 1.715-2.040 QSO J0243-0550 1.805 1.715-1.777 QSO B0241-01 4.053 2.767-4.002 4.023 $\ldots$

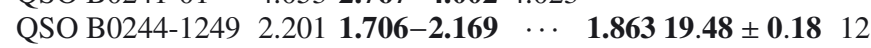
\begin{tabular}{lllll} 
QSO B0253+0058 1.346 & 0 & $\cdots$ & $0.72520 .70 \pm 0.17$ & 11 \\
\hline
\end{tabular} 
Table 1. continued.

\begin{tabular}{|c|c|c|c|c|c|c|}
\hline Quasar & $z_{\mathrm{em}}$ & $z_{\min }-z_{\max }$ & $z_{\text {LLS }}$ & abs & $\begin{array}{c}\log N_{\mathrm{HI}} \\
\mathrm{cm}^{-2}\end{array}$ & \\
\hline SO B0254-404 & 2.280 & 1.708 & $\ldots$ & \multicolumn{2}{|c|}{$2.04620 .45 \pm 0.08$} & 81 \\
\hline & & & $\cdots$ & $\cdots$ & $\cdots$ & \\
\hline 8 & 0.314 & 0 & $\cdots$ & $\cdots$ & $\cdots$ & \\
\hline 2 & 2.096 & $1.515-2.065$ & $\ldots$ & $\ldots$ & $\ldots$ & \\
\hline 7 & 2.130 & 99 & $\ldots$ & $\ldots$ & $\ldots$ & \\
\hline & 2.144 & 1.50 & $\cdots$ & $\cdots$ & $\ldots$ & \\
\hline $\mathrm{B}$ & 2.122 & 1.5 & $\ldots$ & 1.788 & $.00 \pm 0$ & \\
\hline 38 & 1.927 & 1.5 & $\ldots$ & $\ldots$ & $\ldots$ & \\
\hline 5 & 2.423 & 89 & $\cdots$ & $\cdots$ & $\cdots$ & \\
\hline & 2.736 & 1.6 & 2.470 & $\cdots$ & & \\
\hline QSO J0332 & 2.679 & & 2.671 & 2.4112 & 07 & $7 \quad 10$ \\
\hline & & & & 2.6 & & 5 \\
\hline Q & 3.442 & 2.2 & 3.353 & 3.178 & .07 & $7 \quad 18$ \\
\hline 21 & 5.020 & & 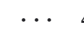 & 4.060 & 20.40 & \\
\hline QSO J0338-0005 & 3.049 & 1.5 & $\cdots$ & 2.2302 & 25 & 51 \\
\hline & & & & 2. & & \\
\hline & 3 & & $\cdots$ & 3.0 & & \\
\hline QSO B0347-383 & 3.22 & & 3.043 & 3.025 & $20.73 \pm 0.05$ & \\
\hline 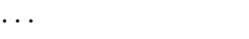 & & 2.397- & $\cdots$ & $\cdots$ & $\cdots$ & \\
\hline$\cdots$ & 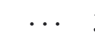 & $3.039-3.181$ & $\ldots$ & $\ldots$ & $\ldots$ & \\
\hline & 2.944 & 0 & $\ldots$ & 1.9472 & $20.30 \pm 0$ & \\
\hline 8 & 1.911 & $5-1.882$ & $\ldots$ & $\ldots$ & $\ldots$ & \\
\hline QSC & 2.823 & $1.715-2.714$ & .. & 1.4052 & $20.18 \pm 0$ & \\
\hline J0401 & 1.507 & 0 & $\ldots$ & $\cdots$ & $\cdots$ & \\
\hline QSO J0403-1703 & 4.2272 & 2.9 & $\cdots$ & $\cdots$ & $\cdots$ & \\
\hline & & $3.801-4.174$ & $\ldots$ & $\ldots$ & $\ldots$ & \\
\hline QSO J0407-4410 & 3.020 & $1.736-1.899$, & 2.647 & 1.9132 & $20.80 \pm$ & 0 \\
\hline$\cdots$ & & 1.927-1.974, & & 2.5512 & $21.15 \pm 0.15$ & 510 \\
\hline$\cdots$ & $\ldots 2$ & $2.048-2.526$ & $\ldots$ & 2.5952 & $21.05 \pm 0.10$ & 0 \\
\hline$\cdots$ & $\cdots$ & $2.566-2.579$ & $\cdots$ & 2.6212 & $20.45 \pm 0.10$ & \\
\hline$\cdots$ & $\ldots$ & 2.612-2.616, & $\ldots$ & $\cdots$ & $\cdots$ & \\
\hline
\end{tabular}

QSO J0422-3844 $3.123 \quad 2.093-3.1203 .1153 .08219 .37 \pm 0.02 \quad 22$

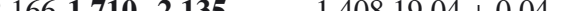
$\begin{array}{lcccccc}\cdots & \cdots & \ldots & \cdots & 1.562 & 19.35 \pm 0.10 & 23 \\ \text { QSO J0430-4855 } & 1.940 & 1.517-1.911 & \cdots & \cdots & \ldots & \end{array}$ QSO B0432-440 2.649 1.715-2.283，․ $2.29720 .78 \pm 0.1021$ $\begin{array}{lcrrrrr}\cdots & \cdots & \mathbf{2 . 3 1 1}-\mathbf{2 . 6 1 3} & \cdots & \cdots & \cdots & \\ \text { QSO B0438-43 } & 2.852 & \mathbf{1 . 5 5 1}-\mathbf{2 . 3 3 3}, & \cdots & 2.347 & 20.78 \pm 0.12 & 21\end{array}$

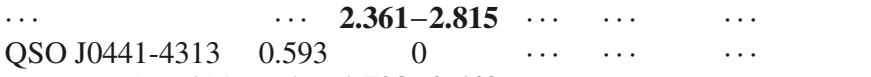
QSO B0449-1645 2.679 1.708-2.643 $\cdots \cdots$ QSO B0450-1310B 2.250 1.517-2.055，․ $2.06720 .50 \pm 0.07 \quad 10$

QSO J0455-4216 PKS 0454-220 4C-02.19

QSO B0512-3329 ‥ 2.079-2.187 $2.6611 .514-2.624$ 0.5340 $2.2861 .550-2.253$ QSO B0515-4414 $1.713 \quad 1.517-1.686$ J051939.8-364613 1.349 QSO B0528-2505

QSO J0530+13 2.765 1.889-2.128, $2.8272 .14120 .95 \pm 0.0526$ QSO B0551-36 ‥ 2.154-2.714 2.070 1.861-2.039 2.318 1.517-1.949, $1.975-2.285$

J060008.1-504036 3.130 2.063-2.138, $3.0802 .14920 .40 \pm 0.126$ 2.160-3.089

QSO B0606-2219 1.926 1.750-1.898

QSO B0642-5038 3.090 1.757-2.644, $2.6702 .65920 .95 \pm 0.0810$ QSO B0736+01 $\quad 0.19$ QSO B0810+2554 1.510 QSO B0827+2421 0.939 2.932-3.050 0
0
0 ... $\quad \cdots \quad \cdots$ QSO B0841+129 2.495 1.707-1.846, $0.51820 .30 \pm 0.0411$ (1.846, $\cdots \quad 1.86421 .00 \pm 0.10 \quad 10$ 1.881-2.362, $\cdots \quad 2.37521 .05 \pm 0.10 \quad 10$
Table 1. continued.

\begin{tabular}{|c|c|c|c|c|c|c|}
\hline uas & $z_{\mathrm{em}}$ & $z_{\min }-z_{\max }$ & $z_{\text {LLS }}$ & $z_{\mathrm{abs}}$ & $\begin{array}{c}\log N_{\mathrm{HI}} \\
\mathrm{cm}^{-2}\end{array}$ & Ref \\
\hline & $\begin{array}{l}\ldots \\
\ldots\end{array}$ & 2 & & & & \\
\hline SO $0908+0603$ & 2.793 & 0 & $\ldots$ & $\ldots$ & ... & \\
\hline $\begin{array}{l}\mathrm{QSC} \\
\ldots\end{array}$ & $\begin{array}{c}2.785 \\
\ldots\end{array}$ & & $\begin{array}{c}2.629 \\
\ldots\end{array}$ & & & \\
\hline & 2.2 & & $\ldots$ & .. & & \\
\hline & 1.6 & & $\ldots$ & $\ldots$ & $\cdots$ & \\
\hline QSC & $\begin{array}{c}2.910 \\
\ldots\end{array}$ & & $\begin{array}{l}\cdots \\
\cdots\end{array}$ & $\begin{array}{c}2.68 \\
\ldots\end{array}$ & $\begin{array}{c}.50 \pm 0.10 \\
\ldots\end{array}$ & \\
\hline $\begin{array}{l}\text { QSO F } \\
\cdots\end{array}$ & $\begin{array}{c}4.369 \\
\ldots\end{array}$ & $\begin{array}{l}2.936-3.846 \\
3.870-4.158\end{array}$ & $\begin{array}{l}\cdots \\
\cdots\end{array}$ & $\begin{array}{l}3.23 \\
3.85\end{array}$ & $\begin{array}{l}20.25= \\
20.60=\end{array}$ & $\begin{array}{c}29 \\
3\end{array}$ \\
\hline $\begin{array}{l}\text {. } \\
\mathrm{PSO} B 0952+179\end{array}$ & 1470 & . & $\begin{array}{l}\cdots \\
\ldots\end{array}$ & $\begin{array}{l}4.203 \\
0238\end{array}$ & $20.55 \pm 0.10$ & 27 \\
\hline
\end{tabular}

QSO B0952-0115 $4.4263 .034-4.3704 .2033 .47620 .04 \pm 0.07 \quad 6$

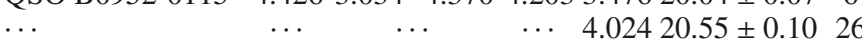

QSO B1005-333 1.837 1.731-1.809

$\begin{array}{lllllll}\text { QSO J1009-0026 } & 1.241 & 0 & \cdots & 0.840 & 20.20 \pm 0.06 & 11\end{array}$

$\begin{array}{llllll}1 . . & \ldots & \ldots & \ldots & 0.88019 .48 \pm 0.08 & 11\end{array}$

$\begin{array}{llllll}\text { LBQS 1026-0045B } 1.530 & 0 & \cdots & 0.632 & 19.95 \pm 0.07 & 7\end{array}$

… $0.70920 .04 \pm 0.06 \quad 7$

QSO B1027+0540 6.311 5.581-6.238

Q1036-272 $\quad 3.090$ 2.096-2.779， ‥ $2.79220 .65 \pm 0.13 \quad 6$

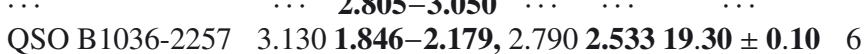

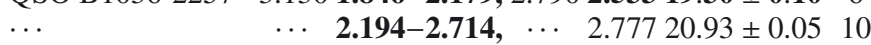
$\begin{array}{lllllll}\ldots & \ldots & \mathbf{2 . 7 9 3}-\mathbf{3 . 0 9 1} & \ldots & \ldots & \ldots\end{array}$

QSO J1039-2719 2.193 1.706-2.132，․ $2.13919 .90 \pm 0.0510$

$\begin{array}{llll}\cdots & \cdots & \mathbf{2 . 1 4 6}-\mathbf{2 . 1 6 3} \\ \text { QSO B1038-2712 } & 2.331 & \mathbf{1 . 5 0 5}-\mathbf{2 . 2 9 8}\end{array}$

$\begin{array}{lllllll}\text { QSO B } 1036-268 & 2.460 & \mathbf{1 . 7 0 7}-\mathbf{2 . 4 2 5} & \cdots & \mathbf{2 . 2 3 5} & \mathbf{1 9 . 9 6} \pm \mathbf{0 . 0 9} & 6\end{array}$

J104540.7-101813 $1.261 \quad 0$

J104642.9+053107 2.682 1.517-2.182

QSO B1044+059 1.226

QSO B1044+056 1.306

QSO B1045+056 1.230

QSO B1052-0004 1.021

QSO B1055-301 2.523 1.552-1.885,

‥ $1.923-2.488$

QSO B1104-181 $2.319 \begin{array}{llllll}1.517-2.286 & \cdots & 1.66120 .85 \pm 0.01 & 30\end{array}$

$\begin{array}{lllllll}\text { QSO J1107+0048 } & 1.392 & 0 & \cdots & 0.740 & 21.00 \pm 0.04 & 11\end{array}$

QSO B1108-07 3.922 2.619-3.598, $3.824 \mathbf{3 . 4 8 2} 19.95 \pm \mathbf{0 . 0 7} 1$

$\begin{array}{lllllll}\cdots & \cdots & \mathbf{3 . 6 1 8}-\mathbf{3 . 8 7 4} & \cdots & 3.608 & 20.37 \pm 0.07 & 1\end{array}$

QSO J1113-1533 3.370 2.241-2.574, $3.2953 .26521 .30 \pm 0.0510$

‥ $\quad \cdots \quad 2.615-3.248, \ldots$

$\ldots \quad \ldots \quad 3.282-3.326$

QSO B1114-220 2.282 0

QSO B1114-0822 4.495 3.180-3.734, $\cdots \quad 4.25820 .02 \pm 0.12 \quad 6$

$\begin{array}{llllll}\cdots & \cdots & 3.804-4.246, & \ldots & \ldots & \ldots\end{array}$

$\begin{array}{lll} & \mathbf{3 . 8 0 4}-\mathbf{4 . 2 4 6} \\ \cdots & \ldots & \mathbf{4 . 2 7 0}-\mathbf{4 . 3 0 2}\end{array}$

$\begin{array}{lllllll}\text { QSO B1122-168 } & 2.400 & 1.706-2.368 & \cdots & 0.682 & 20.45 \pm 0.05 & 31\end{array}$ J112910.9-231628 $1.019 \quad 0$

QSO J1142+2654 2.630 2.095-2.594

QSO B1145-676 $0.210 \quad 0$

QSO B1151+068 2.762 1.517-1.757，‥ $1.77521 .30 \pm 0.0824$ $\begin{array}{lcccccc}\cdots & \cdots & \mathbf{1 . 7 9 3}-\mathbf{2 . 7 2 4} & \ldots & \ldots & \ldots & \\ \mathrm{J} 115538.6+053050 & 3.475 & \mathbf{2 . 2 6 6}-\mathbf{2 . 5 9 7}, 3.350 & 2.608 & 20.37 \pm 0.11 & 6\end{array}$ $\begin{array}{lccccccc}\mathrm{J} 115538.6+053050 & 3.475 & \mathbf{2 . 2 6 6}-\mathbf{2 . 5 9 7}, & 3.350 & 2.608 & 20.37 \pm 0.11 & 6 \\ \ldots & \ldots & \mathbf{2 . 6 1 9}-\mathbf{3 . 2 5 8} & \ldots & 3.327 & 21.00 \pm 0.10 & 6\end{array}$

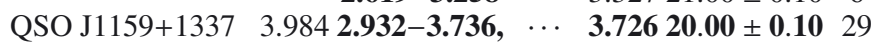
$\begin{array}{lcrrrr}\ldots & \ldots & \mathbf{3 . 8 0 0}-\mathbf{3 . 9 3 5} & \ldots & \ldots & \ldots \\ \text { QSO B1158-1842 } & 2.453 & 1.509-2.418 & \ldots & \ldots & \ldots\end{array}$

QSO B1202-074 4.695 3.129-4.635 $4.5004 .38320 .60 \pm 0.1426$

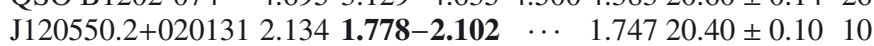

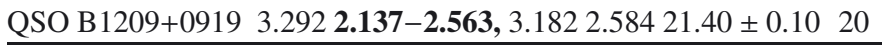


Table 1. continued.

\begin{tabular}{|c|c|c|c|c|c|c|}
\hline Quasar & $z_{\mathrm{em}}$ & $z_{\min }-z_{\max }$ & $z_{\mathrm{LLS}}$ & $z_{\text {abs }}$ & $\underset{\mathrm{cm}^{-2}}{\log N_{\mathrm{HI}}}$ & Ref. \\
\hline$\ldots$ & $\ldots$ & $2.599-3.251$ & $\ldots$ & $\ldots$ & .. & \\
\hline LBQS $1209+1046$ & 2.193 & $1.706-2.161$ & $\ldots$ & 0.633 & 20.30 & 32 \\
\hline $\begin{array}{l}\text { LBQS } 1210+1731 \\
\ldots\end{array}$ & $\begin{array}{c}2.543 \\
\ldots\end{array}$ & $\begin{array}{l}1.517-1.878 \\
1.906-2.187\end{array}$ & $\begin{array}{l}\cdots \\
\cdots\end{array}$ & $\begin{array}{l}1.8922 \\
\ldots\end{array}$ & $\begin{array}{c}20.70 \pm 0.08 \\
\ldots\end{array}$ & 310 \\
\hline QSO J1215+3309 & 0.616 & 0 & $\cdots$ & $\cdots$ & $\ldots$ & \\
\hline $\begin{array}{l}\text { QSO B1220-1800 } \\
\ldots\end{array}$ & $\begin{array}{c}2.160 \\
\ldots\end{array}$ & $\begin{array}{l}1.698-2.102 \\
2.122-2.130\end{array}$ & $\begin{array}{l}\cdots \\
\cdots\end{array}$ & $\begin{array}{c}2.1122 \\
\ldots\end{array}$ & $\begin{array}{c}20.12 \pm 0.07 \\
\ldots\end{array}$ & 710 \\
\hline LBQS $1223+1753$ & 2.940 & 2.044-2.446, & 2.941 & 2.466 & $21.40 \pm 0.10$ & 10 \\
\hline$\cdots$ & $\cdots$ & $2.488-2.901$ & $\cdots$ & 2.557 & $19.32 \pm 0.15$ & 533 \\
\hline QSO B1228-113 & 3.528 & 1.715-2.180, & $\cdots$ & 2.1932 & $20.60 \pm 0.10$ & 21 \\
\hline$\cdots$ & $\cdots$ & $2.206-2.718$ & $\cdots$ & $\cdots$ & $\cdots$ & \\
\hline$\cdots$ & $\ldots$ & $2.932-3.485$ & $\cdots$ & $\ldots$ & $\ldots$ & \\
\hline $\begin{array}{l}\text { QSO B1230-101 } \\
\ldots\end{array}$ & $\begin{array}{c}2.394 \\
\ldots\end{array}$ & $\begin{array}{l}1.805-1.919 \\
1.943-2.361\end{array}$ & $\begin{array}{l}\cdots \\
\cdots\end{array}$ & $\begin{array}{c}1.9312 \\
\ldots\end{array}$ & $\begin{array}{c}20.48 \pm 0.10 \\
\ldots\end{array}$ & 21 \\
\hline LBQS $1232+0815$ & 2.570 & 1.704-2.327, & $\cdots$ & 1.720 & $19.48 \pm 0.13$ & 36 \\
\hline$\cdots$ & $\cdots$ & $2.350-2.535$ & $\cdots$ & 2.334 & $20.90 \pm 0.08$ & 34 \\
\hline LBQS $1242+0006$ & 2.084 & $1.706-1.812$ & $\cdots$ & 1.8242 & $20.45 \pm 0.10$ & 10 \\
\hline & & $1.836-2.053$ & $\cdots$ & $\cdots$ & $\cdots$ & \\
\hline QSO J1246-0730 & 1.286 & 0 & $\cdots$ & $\ldots$ & $\ldots$ & \\
\hline LBC & 2.117 & $1.842-2.088$ & $\ldots$ & 1.7812 & $21.45 \pm 0.15$ & 535 \\
\hline $\mathrm{J} 124957.2$ & 3.635 & $2.414-3.589$ & 3.550 & $\cdots$ & $\cdots$ & \\
\hline QSO B1249-02 & 1.192 & 0 & $\cdots$ & $\cdots$ & $\cdots$ & \\
\hline QSO B1256-177 & 1.956 & $1.789-1.926$ & $\cdots$ & $\cdots$ & $\cdots$ & \\
\hline QSO J1306+0356 & 5.999 & $4.511-5.929$ & $\cdots$ & $\cdots$ & $\cdots$ & \\
\hline QSO B1317-0507 & 3.710 & $1.911-3.663$ & 2.880 & $\cdots$ & $\cdots$ & \\
\hline QSO B1318-263 & 2.027 & $1.715-1.997$ & $\ldots$ & $\ldots$ & $\cdots$ & \\
\hline LBQS 1320-0006 & 1.388 & 0 & $\cdots$ & 0.716 & $20.54 \pm 0.15$ & 511 \\
\hline QSC & 1.882 & $1.706-1.853$ & $\cdots$ & $\cdots$ & $\cdots$ & \\
\hline QSO J1330-2522 & 3.910 & $2.547-2.714$ & $\cdots$ & 2.654 & $19.56 \pm 0.13$ & 36 \\
\hline$\cdots$ & $\cdots$ & 2.932-3.736, & $\cdots$ & 2.910 & 20.00 & 3 \\
\hline$\cdots$ & $\cdots$ & $3.800-3.861$ & $\cdots$ & 3.080 & $19.88 \pm 0.09$ & 9 3 \\
\hline $\begin{array}{l}\text { QSO B1331+170 } \\
\ldots\end{array}$ & $\begin{array}{c}2.084 \\
\ldots\end{array}$ & $\begin{array}{l}1.517-1.718 \\
1.801-2.053\end{array}$ & $\begin{array}{l}\cdots \\
\cdots\end{array}$ & $\begin{array}{c}1.7762 \\
\ldots\end{array}$ & $\begin{array}{c}21.15 \pm 0.07 \\
\ldots\end{array}$ & 710 \\
\hline QSO & 3.212 & -3.092 & 3.152 & 3.118 & $20.05 \pm 0.08$ & 310 \\
\hline QSC & 2.134 & $1.512-2.103$ & $\cdots$ & $\ldots$ & $\ldots$ & \\
\hline QSO B1347-2457 & 2.578 & $1.512-2.542$ & $\cdots$ & $\cdots$ & $\cdots$ & \\
\hline QSO J1356-1101 & 3.006 & $2.090-2.493$ & $\cdots$ & 2.397 & $19.85 \pm 0.08$ & 36 \\
\hline$\cdots$ & $\cdots$ & $2.510-2.924$ & $\cdots$ & 2.5012 & $20.44 \pm 0.05$ & 527 \\
\hline$\cdots$ & $\cdots$ & $\cdots$ & $\cdots$ & 2.9672 & $20.80 \pm 0.10$ & 10 \\
\hline QSO B & 2.522 & $2.093-2.487$ & $\ldots$ & $\ldots$ & $\cdots$ & \\
\hline QSO B1409+0930 & 2.838 & $1.761-2.008$ & 2.680 & 2.0192 & $20.65 \pm 0.10$ & 10 \\
\hline$\cdots$ & $\cdots$ & $2.030-2.448$ & $\cdots$ & 2.456 & $20.53 \pm 0.08$ & 810 \\
\hline$\cdots$ & $\cdots$ & $2.463-2.802$ & $\cdots$ & 2.668 & $19.80 \pm 0.08$ & 310 \\
\hline QSO B1412-096 & 2.001 & $1.715-1.971$ & $\cdots$ & $\cdots$ & $\cdots$ & \\
\hline QSO J1421-0643 & 3.689 & $2.357-3.096$ & 3.474 & 3.448 & $20.40 \pm 0.10$ & 18 \\
\hline QSO B1424-41 & 1.522 & 0 & $\cdots$ & $\cdots$ & $\cdots$ & \\
\hline QSO B1429-008B & 2.082 & $1.739-2.052$ & $\cdots$ & $\cdots$ & $\cdots$ & \\
\hline $\begin{array}{l}\text { QSO J1439+1117 } \\
\ldots\end{array}$ & $\begin{array}{c}2.583 \\
\ldots\end{array}$ & $\begin{array}{l}1.715-2.409 \\
2.427-2.547\end{array}$ & $\begin{array}{l}\cdots \\
\cdots\end{array}$ & $\begin{array}{l}2.418 \\
\ldots\end{array}$ & $\begin{array}{c}20.10 \pm 0.10 \\
\ldots\end{array}$ & 36 \\
\hline QSO J1443+2724 & 4.443 & 2.932-3.736, & $\cdots$ & 4.224 & $20.95 \pm 0.08$ & 327 \\
\hline$\cdots$ & .. & $3.802-4.209$ & $\cdots$ & $\cdots$ & $\cdots$ & \\
\hline$\cdots$ & $\cdots$ & $4.242-4.384$ & $\cdots$ & $\cdots$ & $\cdots$ & \\
\hline LBQS 1444+0126 & 2.210 & 1.710-2.082, & $\cdots$ & 2.087 & $20.25 \pm 0.07$ & 710 \\
\hline$\cdots$ & . & $2.091-2.178$ & $\cdots$ & $\cdots$ & $\cdots$ & \\
\hline QS & 2.215 & $1.509-2.184$ & $\cdots$ & $\cdots$ & $\cdots$ & \\
\hline J145 & 4.763 & $3.103-4.705$ & 4.369 & $\cdots$ & $\cdots$ & \\
\hline QSO J1453+0029 & 1.297 & 0 & $\cdots$ & $\cdots$ & $\cdots$ & \\
\hline $\mathrm{J} 151352.52+085555$ & 2.904 & $1.745-2.865$ & $\cdots$ & $\cdots$ & $\cdots$ & \\
\hline QSO J1621-0042 & 3.700 & $2.110-3.655$ & 3.138 & 3.104 & $19.70 \pm 0.20$ & 37 \\
\hline 4C 12.59 & 1.792 & $1.517-1.764$ & $\cdots$ & 0.5312 & $20.70 \pm 0.09$ & 9 11 \\
\hline$\cdots$ & $\cdots$ & $\cdots$ & $\cdots$ & 0.900 & $19.70 \pm 0.04$ & 411 \\
\hline QSO J1723+2243 & 4.520 & 3.072-3.687, & $\cdots$ & 3.6972 & $20.35 \pm 0.10$ & 29 \\
\hline$\cdots$ & $\cdots$ & 3.707-3.736, & $\cdots$ & 4.155 & $19.23 \pm 0.12$ & 26 \\
\hline$\cdots$ & $\cdots$ & $3.802-4.464$ & $\cdots$ & $\cdots$ & $\cdots$ & \\
\hline QSO B1730-130 & 0.902 & 0 & $\cdots$ & $\ldots$ & $\cdots$ & \\
\hline
\end{tabular}

Table 1. continued.

\begin{tabular}{|c|c|c|c|c|c|c|}
\hline Quasar & $z_{\mathrm{em}}$ & $z_{\min }-z_{\max }$ & $z_{\text {LLS }}$ & $z_{\mathrm{abs}}$ & $\begin{array}{c}\log N_{\mathrm{HI}} \\
\mathrm{cm}^{-2} \\
\end{array}$ & Ref. \\
\hline QSO B1741-038 & 1.054 & 0 & $\ldots$ & $\ldots$ & $\ldots$ & \\
\hline QSO B1937-1009 & 3.787 & $2.932-3.738$ & $\ldots$ & $\ldots$ & $\ldots$ & \\
\hline QSO B 1935-692 & 3.152 & $2.932-3.110$ & $\ldots$ & $\ldots$ & $\ldots$ & \\
\hline QSO B2000-330 & 3.783 & $2.422-3.735$ & 3.560 & 3.172 & $19.80 \pm 0.25$ & 37 \\
\hline$\cdots$ & $\ldots$ & $\ldots$ & $\cdots$ & 3.188 & $.80 \pm 0.15$ & 37 \\
\hline$\cdots$ & $\cdots$ & $\cdots$ & $\cdots$ & 3.192 & $19.20 \pm 0.25$ & 37 \\
\hline QSO J2107-0620 & 0.642 & 0 & $\ldots$ & $\ldots$ & $\ldots$ & \\
\hline LBQS 2113-4345 & 2.053 & $1.510-2.021$ & $\ldots$ & $\ldots$ & $\ldots$ & \\
\hline LBQS 2114-4347 & 2.040 & $1.509-2.010$ & $\ldots$ & 1.912 & $19.50 \pm 0.10$ & 6 \\
\hline $\mathrm{J} 211739.5-433538$ & 2.050 & $1.509-2.020$ & $\ldots$ & $\ldots$ & . & \\
\hline $\begin{array}{l}\text { QSO J2119-3536 } \\
\ldots\end{array}$ & $\begin{array}{c}2.341 \\
\ldots\end{array}$ & $\begin{array}{l}1.710-1.992 \\
2.000-2.308\end{array}$ & $\begin{array}{l}\cdots \\
\cdots\end{array}$ & $\begin{array}{l}1.996 \\
\ldots\end{array}$ & $\begin{array}{c}.10 \pm 0.07 \\
\ldots\end{array}$ & 27 \\
\hline QSO B2126-15 & 3.268 & $1.838-3.225$ & 2.783 & 2.638 & $19.25 \pm 0.15$ & 37 \\
\hline & $\cdots$ & $\cdots$ & $\cdots$ & 2.769 & $19.20 \pm 0.15$ & 37 \\
\hline QSO B2129-4653 & 2.230 & $1.706-2.198$ & $\ldots$ & $\cdots$ & $\cdots$ & \\
\hline J213314.2-464031 & 2.208 & $1.730-2.176$ & $\cdots$ & $\cdots$ & $\cdots$ & \\
\hline $\begin{array}{l}\text { LBQS 2132-4321 } \\
\ldots\end{array}$ & $\begin{array}{c}2.420 \\
\ldots\end{array}$ & $\begin{array}{l}1.710-1.907 \\
1.923-2.383\end{array}$ & $\begin{array}{l}\cdots \\
\cdots\end{array}$ & $\begin{array}{l}1.916 \\
\ldots\end{array}$ & $\begin{array}{c}.74 \pm 0.09 \\
\ldots\end{array}$ & 6 \\
\hline LBQS 2138-4427 & 3.170 & 2.090-2.371, & $\ldots$ & 2.383 & $20.60 \pm 0.05$ & 1 \\
\hline$\cdots$ & & 2.395-2.837, & $\ldots$ & 2.852 & $0.98 \pm 0.05$ & 27 \\
\hline$\cdots$ & $\ldots$ & 2.867-3.099 & $\ldots$ & $\ldots$ & $\ldots$ & \\
\hline QSO B2139-4433 & 3.220 & $2.932-3.178$ & $\ldots$ & $\ldots$ & $\ldots$ & \\
\hline QSO B2149-306 & 2.345 & $1.715-2.312$ & $\ldots$ & $\cdots$ & $\ldots$ & \\
\hline QSO B2204-408 & 3.155 & 2.932-3.113 & $\ldots$ & $\ldots$ & $\ldots$ & \\
\hline LBQS 2206-1958A & 2.560 & $1.517-1.912$ & $\ldots$ & 1.921 & $20.67 \pm 0.05$ & 30 \\
\hline$\cdots$ & & 1.932-2.071, & $\ldots$ & 2.076 & $20.44 \pm 0.05$ & 10 \\
\hline$\cdots$ & & $2.083-2.526$ & $\ldots$ & $\cdots$ & $\ldots$ & \\
\hline QSO J & 1.476 & 0 & $\ldots$ & $\cdots$ & $\cdots$ & \\
\hline QSO J2220-2803 & 2.406 & $1.517-2.373$ & $\cdots$ & $\cdots$ & $\cdots$ & \\
\hline QSO B2222-396 & 2.198 & $1.706-2.114$ & $\cdots$ & 2.154 & $20.85 \pm 0.10$ & 10 \\
\hline QSO J2227-2243 & 1.891 & $1.517-1.863$ & $\cdots$ & $\ldots$ & $\cdots$ & \\
\hline QSO B2225-4025 & 2.030 & $1.716-1.920$ & $\ldots$ & 1.96 & $20.65 \pm 0.10$ & 10 \\
\hline $\begin{array}{l}\text { LBQS } 2230+0232 \\
\ldots\end{array}$ & $\begin{array}{c}2.147 \\
\ldots\end{array}$ & $\begin{array}{l}1.517-1.849 \\
1.879-2.116\end{array}$ & $\begin{array}{l}\cdots \\
\cdots\end{array}$ & $\begin{array}{c}1.864 \\
\ldots\end{array}$ & $\begin{array}{l}90 \pm 0.10 \\
\ldots\end{array}$ & 10 \\
\hline J223851.0-295301 & 2.387 & $1.535-2.356$ & $\ldots$ & $\ldots$ & $\ldots$ & \\
\hline J223922.9-294947 & 1.849 & $1.564-1.821$ & $\cdots$ & $\ldots$ & $\ldots$ & \\
\hline J223938.9-295451 & 1.907 & $1.535-1.878$ & $\ldots$ & $\ldots$ & $\ldots$ & \\
\hline J223941.8-294955 & 2.102 & $1.656-2.071$ & $\ldots$ & 1.825 & $19.84 \pm 0.14$ & 6 \\
\hline J223948.7-294748 & 2.068 & $1.517-2.037$ & $\cdots$ & $\cdots$ & $\ldots$ & \\
\hline J223951.9-294837 & 2.121 & $1.525-2.090$ & $\cdots$ & $\ldots$ & $\ldots$ & \\
\hline QSO B2237-0607 & 4.558 & 2.894-3.735, & $\cdots$ & 4.079 & $20.55 \pm 0.10$ & 4 \\
\hline$\cdots$ & $\cdots$ & $3.802-4.068$ & $\cdots$ & $\cdots$ & $\cdots$ & \\
\hline$\cdots$ & ... & $4.090-4.500$ & $\cdots$ & $\cdots$ & .. & \\
\hline QSO J2247-1237 & 1.892 & $1.716-1.863$ & $\cdots$ & $\cdots$ & . & \\
\hline $\begin{array}{l}\text { QSO B2311-373 } \\
\ldots\end{array}$ & $\begin{array}{c}2.476 \\
\ldots\end{array}$ & $\begin{array}{l}1.706-2.171, \\
2.193-2.443\end{array}$ & $\begin{array}{l}\cdots \\
\cdots\end{array}$ & $\begin{array}{c}2.182 \\
\ldots\end{array}$ & $\begin{array}{c}20.48 \pm 0.13 \\
\ldots\end{array}$ & 10 \\
\hline J232046.7-294406 & 2.401 & $1.509-2.367$ & .. & $\ldots$ & $\ldots$ & \\
\hline J232059.4-295520 & 2.317 & $1.517-2.284$ & $\cdots$ & $\cdots$ & $\cdots$ & \\
\hline J232114.3-294725 & 2.677 & $2.091-2.640$ & $\cdots$ & $\cdots$ & $\cdots$ & \\
\hline J232121.2-294350 & 2.184 & $1.620-2.152$ & $\ldots$ & $\ldots$ & $\ldots$ & \\
\hline QSO B2318-1107 & 2.960 & 1.509-1.618, & $\ldots$ & 1.629 & $20.52 \pm 0.14$ & 6 \\
\hline$\ldots$ & $\ldots$ & 1.640-1.977, & $\ldots$ & 1.989 & $20.68 \pm 0.05$ & 10 \\
\hline$\cdots$ & $\ldots$ & 2.001-2.714, & $\cdots$ & $\cdots$ & $\cdots$ & \\
\hline$\ldots$ & & $2.932-2.920$ & $\ldots$ & .. & $\cdots$ & \\
\hline QSO J2328+0022 & 1.302 & 0 & $\cdots$ & 0.652 & $20.32 \pm 0.07$ & 11 \\
\hline QSO B2332-094 & 3.330 & 2.177-3.045, & 3.235 & 2.287 & $20.07 \pm 0.07$ & 8 \\
\hline .. & $\ldots$ & $3.069-3.289$ & $\ldots$ & 3.057 & $20.50 \pm 0.07$ & 10 \\
\hline $\mathrm{J} 233544.2+150118$ & 0.791 & 0 & $\cdots$ & 0.680 & $19.70 \pm 0.30$ & 11 \\
\hline QSO B2342+3417 & 3.010 & 2.091-2.891, & $\ldots$ & 2.909 & $21.10 \pm 0.10$ & 10 \\
\hline$\cdots$ & $\cdots$ & $2.927-2.970$ & $\ldots$ & 2.940 & $20.18 \pm 0.10$ & 6 \\
\hline QSO J2346+1247 & 2.578 & $1.715-2.538$ & .. & 2.569 & $20.98 \pm 0.04$ & 38 \\
\hline QSO B $2343+125$ & 2.763 & $1.601-2.185$ & 2.465 & 2.431 & $20.40 \pm 0.07$ & 10 \\
\hline QSO B2345+000 & 2.654 & $1.715-2.617$ & .. & $\cdots$ & $\cdots$ & \\
\hline QSO B2347-4342 & 2.885 & $1.812-2.846$ & 2.750 & $\ldots$ & ... & \\
\hline
\end{tabular}


Table 1. continued.

\begin{tabular}{|c|c|c|c|c|c|c|}
\hline Quasar & $z_{\mathrm{em}}$ & $z_{\min }-z_{\max }$ & $z_{\text {LLS }}$ & $z_{\mathrm{abs}}$ & $\underset{\mathrm{cm}^{-2}}{\log N_{\mathrm{HI}}}$ & Ref. \\
\hline QSO B2348-0180 & 3.023 & 1.962-2.032, & 2.946 & 2.426 & $20.50 \pm 0.10$ & 27 \\
\hline$\cdots$ & $\cdots$ & 2.069-2.415, & $\cdots$ & 2.6152 & $21.30 \pm 0.08$ & 10 \\
\hline$\cdots$ & $\cdots$ & 2.437-2.596, & $\cdots$ & $\cdots$ & $\cdots$ & \\
\hline$\cdots$ & $\cdots$ & 2.634-2.718, & $\ldots$ & $\ldots$ & $\ldots$ & \\
\hline$\ldots$ & $\ldots$ & $2.932-2.982$ & $\ldots$ & $\ldots$ & $\ldots$ & \\
\hline QSO B2348-147 & 2.933 & 1.838-2.264, & $\cdots$ & 2.2792 & $20.56 \pm 0.08$ & 10 \\
\hline$\ldots$ & $\ldots$ & $2.288-2.894$ & $\ldots$ & $\ldots$ & $\ldots$ & \\
\hline J235534.6- & 1.579 & 0 & $\cdots$ & $\cdots$ & $\cdots$ & \\
\hline $\mathrm{J} 235702.5-004824$ & 3.013 & $2.002-2.973$ & 3.000 & 2.4792 & $20.41 \pm 0.08$ & 6 \\
\hline QSO J2359-1241 & 0.868 & 0 & $\cdots$ & $\cdots$ & $\cdots$ & \\
\hline
\end{tabular}

\section{Appendix A: Description of the individual sightlines}

This appendix describes the individual sightlines of all the quasars with $z_{\mathrm{em}}>1.5$ presented in Table 1 . For each of them, we have examined the abstract of the observing proposal to determine if any knowledge of the sightline characteristics were known prior to the execution of the proposal, which may represent a bias for our statistical study of sub-DLAs. We provide here a short description of the original observing aims and a description of our decision on whether to include or exclude the redshift path and/or the sub-DLAs along these sightlines.

- LBQS 2359-01216B was observed with the aim of measuring the molecular and metal content of known DLAs. The redshift path was included in our statistical sample to search for unknown sub-DLAs.

- QSO J0003-2323 was observed as part of the ESO Large Program for IGM studies. The redshift path and an additional sub-DLA reported by Richter et al. (2005) were excluded from the statistical sample.

- QSO B0002-422 was observed as part of the ESO Large Program for IGM studies. The redshift path was excluded from the statistical sample.

- QSO 0006-6208 was targeted in a search for new sub-DLAs. Therefore, the redshift path and a sub-DLA previously reported by Péroux et al. (2005) were included in the statistical sample.

- QSO J0008-0958 was observed with the aim of measuring the metal content of a known DLA. The redshift path was included in our statistical sample to search for unknown sub-DLAs.

- QSO J0008-2900 was observed in a study of the tomography of the IGM. Therefore, the redshift path and an additional sub-DLA reported in Paper I were excluded from the statistical sample.

- QSO J0008-2901 was observed in a study of the tomography of the IGM. Therefore, the redshift path and an additional sub-DLA reported in Paper I were excluded from the statistical sample.

- QSO B0008+006 was observed in a study of its BAL features and therefore the redshift path was included in our statistical sample to search for unknown sub-DLAs.

- LBQS 0009-0138 was targeted in a study the metal content of sub-DLAs at $0.7<z<1.5$. The redshift path was included to search for sub-DLAs at higher redshift.

- LBQS 0010-0012 was observed with the aim of measuring the molecular content of a known DLA. The redshift path was included in our statistical sample to search for unknown sub-DLAs.
- LBQS 0013-0029 was observed with the aim of measuring the molecular content of a known DLA. The redshift path was included in our statistical sample to search for unknown sub-DLAs. The sub-DLA found by Ledoux et al. (2003) was not included in our redshift path because it is near the known DLA.

- QSO B0027-1836 was observed in a search for molecules in a known DLA. The redshift path was included in our statistical sample to search for unknown sub-DLAs.

- J004054.7-091526 was observed in a study of the intrinsic properties of the quasar. Therefore, the redshift path and an additional sub-DLA found by Noterdaeme et al. (2009) were included.

- QSO J0041-4936 was observed with the aim of measuring the deuterium content of a known DLA. The redshift path was included in our statistical sample to search for unknown sub-DLAs.

- QSOB0039-407 was targeted in a study the metal content of $\mathrm{Mg}$ II absorbers at $0.6<z<1.5$. The redshift path was included to search for sub-DLAs at higher redshift.

- QSO B0039-3354 was observed with the aim of measuring the metal content of known DLAs. The redshift path was included in our statistical sample to search for unknown sub-DLAs.

- LBQS 0041-2638 was observed in a study of the tomography of the IGM. Therefore, the redshift path was excluded from the statistical sample.

- LBQS 0041-2707 was observed in a study of the tomography of the IGM. Therefore, the redshift path was excluded from the statistical sample.

- QSO B0042-2656 was targeted in a study of the tomography of the IGM. Therefore, the redshift path was excluded from the statistical sample.

- LBQS 0042-2930 was observed with the aim of measuring the metal content of known DLAs. The redshift path was included in our statistical sample to search for unknown sub-DLAs.

- LBQS B0042-2657 was targeted in a study of the tomography of the IGM. Therefore, the redshift path was excluded from the statistical sample.

- J004612.2-293110 was targeted in a study of a dark clump at $0.1<z<0.3$ along the line of sight to the quasar. Therefore, the redshift path was included in our statistical sample to search for unknown sub-DLAs.

- LBQS 0047-2538 was observed in a study of the halo of a nearby galaxy along the quasar sightline. Hence, the redshift path was included in our statistical sample to search for unknown sub-DLAs.

- LBQS 0048-2545 was observed in a study of the halo of a nearby galaxy along the quasar sightline. Therefore, the redshift path was included in our statistical sample to search for unknown sub-DLAs.

- QSO B0018-2608 was targeted in a study of the halo of a nearby galaxy along the quasar sightline. Hence, the redshift path was included in our statistical sample to search for unknown sub-DLAs.

- QSOB0055-26 was observed in a study of the IGM. Therefore, the redshift path was excluded from the statistical sample.

- QSO B0058-292 was observed with the aim of measuring the molecular and metal content of a known DLA. The redshift path was included in our statistical sample to search for unknown sub-DLAs. 
- LBQS 0059-2735 was observed in a study of quasar outflows. Therefore, the redshift path was included in our statistical sample to search for unknown sub-DLAs.

- QSO 0100+1300 was targeted in a study of the variation of the fine-structure constant in a quasar with known metal lines. Hence, the redshift path was excluded from the statistical sample.

- QSO J0105-1846 was observed in a study of the metal content of a known DLA. Therefore the redshift path and an additional sub-DLA reported by Fox et al. (2009) were included in our statistical sample.

- QSO B0102-2931 was observed in a study of the correlation function of the IGM. Therefore, the redshift path was excluded from the statistical sample.

- QSO B0103-260 was targeted in a study of the IGM. Hence, the redshift path was excluded from the statistical sample.

- QSO B0109-353 was observed as part of the ESO Large Program for IGM studies. The redshift path was excluded from the statistical sample.

- QSO B0112-30 was observed with the aim of measuring the molecular content of known DLAs. The redshift path was included in our statistical sample to search for unknown sub-DLAs.

- QSO J0124+0044 was targeted with the aim of searching for new sub-DLAs. Therefore, the redshift path and sub-DLAs previously reported by Péroux et al. (2005) were included in the statistical sample.

- QSO B0122-379 was observed as part of the ESO Large Program for IGM studies. The redshift path was excluded from the statistical sample.

- QSO B0122-005 was targeted in a study of the metal content of $\mathrm{Mg}$ II absorbers at $0.6<z<1.5$. The redshift path and an additional sub-DLA reported by Ellison \& Lopez (2009) were included in our statistical sample.

- QSO B0128-2150 was observed with the aim of measuring the deuterium content of $z_{\mathrm{abs}}=1.85$ and $1.64 \mathrm{HI}$ systems. The redshift path was included in our statistical sample to search for unknown sub-DLAs. The $N_{\mathrm{H} \text { I }}$ of the two systems mentioned above are fitted for the first time in Paper I. Only one of them is classified as a sub-DLA, the other system has a lower H I column density.

- QSO B0130-403 was observed in a study of a nearby galaxy along the quasar sightline. Hence, the redshift path was included in our statistical sample to search for unknown sub-DLAs.

- QSO J0133+0400 was targeted in a search of new subDLAs and in a study of the metal content of known DLAs. Therefore, the redshift path and sub-DLAs previously reported by Péroux et al. (2005) were included in the statistical sample.

- QSO B0135-42 was observed in a search of new sub-DLAs. Therefore, the redshift path and sub-DLAs previously reported by Péroux et al. (2005) were included in the statistical sample

- QSO J0139-0824 was targeted in a study of the variation of the fine-structure constant in a quasar with known metal lines. Hence, the redshift path wss excluded from the statistical sample.

- QSO J0143-317 was observed in a study of the IGM. Hence, the redshift path was excluded from the statistical sample.

- QSO J0153-4311 was observed as part of the ESO Large Program for IGM studies. The redshift path was excluded from the statistical sample.
- QSO B0201+113 was observed in a study of the metal content of a known DLA. Therefore, the redshift path was included in our statistical sample to search for unknown sub-DLAs.

- QSO J0209+0517 was observed in a search of new subDLAs. Therefore, the redshift path and a sub-DLA previously reported by Péroux et al. (2005) were included in the statistical sample.

- J021741.8-370100 was observed with the aim of measuring the metal content of known DLAs. The redshift path was included in our statistical sample to search for unknown sub-DLAs.

- QSO B0216+0803 was observed with the aim of measuring the molecular and metal content of a known DLA between $z=2-3$. The redshift path and an additional sub-DLA at lower redshift reported by Dessauges-Zavadsky et al. (2009) were included in our statistical sample.

- QSO B0227-369 was targeted in a study of the metal content of $\mathrm{Mg}$ II absorbers at $0.6<z<1.5$. The redshift path was included to search for sub-DLAs at higher redshift.

- QSO B0237-2322 was targeted as part of the ESO Large Program for IGM studies. The redshift path and sub-DLAs were excluded from the statistical sample.

- QSO J0242+0049 was observed in a study of its BAL features and therefore the redshift path was included in our statistical sample to search for unknown sub-DLAs.

- QSO J0243-0550 was observed in a study of the metal content of Mg II absorbers at $0.6<z<1.5$. The redshift path was included to search for sub-DLAs at higher redshift.

- QSO B0241-01 was observed in a search of new sub-DLAs. The redshift path was included in the statistical sample.

- QSO B0244-1249 was targeted in a study of the metal content of Mg II absorbers at $0.6<z<1.5$. The redshift path and an additional sub-DLA reported by Ellison \& Lopez (2009) were included in the statistical sample.

- QSO B0254-404 was observed with the aim of measuring the metal content of a known DLA. The redshift path was included in our statistical sample to search for unknown sub-DLAs.

- J030640.8-301032 was observed in a study of the tomography of the IGM. Therefore, the redshift path was excluded from the statistical sample.

- J030643.7-301107 was observed in a study of the tomography of the IGM. Hence, the redshift path was excluded from the statistical sample.

- QSO B0307-195A was observed in a study of the tomography of the IGM. Hence, the redshift path was excluded from the statistical sample.

- QSO B0307-195B was observed in a study of the tomography of the IGM. The redshift path and an additional subDLA reported by D'Odorico et al. (2002) were excluded from the statistical sample.

- J031856.6-060038 was observed in a study of the quasar outflows. Therefore, the redshift path was included in our statistical sample to search for unknown sub-DLAs.

- QSO B0329-385 was observed as part of the ESO Large Program for IGM studies. The redshift path was excluded from the statistical sample.

- QB0329-2534 was observed as part of the ESO Large Program for IGM studies. The redshift path was excluded from the statistical sample.

- QSO J0332-4455 was targeted with the aim of measuring the metal content of known DLAs. The redshift path was included in our statistical sample. An additional sub-DLA 
reported by Fox et al. (2009) was excluded because it is $3000 \mathrm{~km} \mathrm{~s}^{-1}$ blueward of the quasar emission line.

- QSO B0335-122 was observed with the aim of measuring the metal content of known DLAs. Therefore, the redshift path was included in our statistical sample to search for unknown sub-DLAs.

- QSO J0338+0021 was observed in a study of the intrinsic properties of the quasar. Therefore, the redshift path was included in our statistical sample to search for unknown sub-DLAs.

- QSOJ0338-0005 was observed in a study of the variation of the fine-structure constant in a quasar with known metal lines. Hence, the redshift path was excluded from the statistical sample.

- QSO B0336-017 was observed in a study of the metal content of a known DLA. Therefore, the redshift path was included in our statistical sample to search for unknown sub-DLAs.

- QSO B0347-383 was observed in a study of the metal content of a known DLA. Therefore, the redshift path was included in our statistical sample to search for unknown sub-DLAs.

- J035320.2-231418 was targeted in a study of the metal content of $\mathrm{Mg}$ II absorbers at $0.6<z<1.5$. The redshift path was included to search for sub-DLAs at higher redshift.

- QSO J0354-2724 was observed in a study of the metal content of sub-DLAs at $0.7<z<1.5$. The redshift path was included to search for sub-DLAs at higher redshift.

- QSO J0403-1703 was observed in a study of narrow absorption lines associated with the quasar. Therefore, the redshift path was included in our statistical sample to search for unknown sub-DLAs.

- QSO J0407-4410 was observed with the aim of measuring the molecular and metal content of known DLAs. The redshift path was included in our statistical sample to search for unknown sub-DLAs.

- QSO J0422-3844 was targeted as part of the ESO Large Program for IGM studies. The redshift path and a sub-DLA were excluded from our statistical sample.

- QSO J0427-1302 was targeted in a study of the metal content of sub-DLAs at $0.7<z<1.5$. The redshift path was included to search for sub-DLAs at higher redshift.

- QSO J0430-4855 was targeted in a study of the IGM. The redshift path was excluded from the statistical sample.

- QSO B0432-440 was observed with the aim of measure the metal content of a known DLA. Therefore, the redshift path was included in our statistical sample to search for unknown sub-DLAs.

- QSO B0438-43 was observed with the aim of measuring the metal content of a known DLA. Therefore, the redshift path was included in our statistical sample to search for unknown sub-DLAs.

- QSO B0449-1645 was targeted in a study of the metal content of a DLA at $z \leq 1.0$. The redshift path was included to search for sub-DLAs at higher redshift.

- QSO B0450-1310B was observed in a study of the metal content of DLAs at $z_{\mathrm{abs}} \gtrsim 2$. The redshift path was included in the statistical sample to search for unknown sub-DLAs.

- QSO J0455-4216 was observed as part of the ESO Large Program for IGM studies. The redshift path was excluded from the statistical sample.

- 4C-02.19 was observed with the aim of measuring the molecular and metal content of a known DLA. Later the quasar was re-observed in a study of the variation of the fine- structure constant in known metal lines. To be conservative, the redshift path was excluded from our statistical sample.

- QSO B0515-4414 was targeted in a study of the IGM. Hence, the redshift path and a lower redshift sub-DLA were excluded from the statistical sample.

- QQSO B0528-2505 was observed with the aim of measuring the molecular and metal content of known DLAs and to constrain the variation of ratio of the electron to proton masses. The redshift path was included in our statistical sample to search for unknown sub-DLAs.

- QSO J0530+13 was targeted in a study of the Mg II absorber at $z=0.79$. The redshift path was included to search for subDLAs at higher redshift.

- QSO B0551-36 was observed with the aim of measuring the molecular content of a known DLA. The redshift path was included in our statistical sample to search for unknown sub-DLAs.

- J060008.1-504036 was observed in a search of deuterium in a known LLS. The redshift path was included in our statistical sample to search for unknown sub-DLAs.

- QSO B0606-2219 was targeted in a study of the metal content of Mg II absorbers at $0.6<z<1.5$. The redshift path wss included to search for sub-DLAs at higher redshift.

- QSO B0642-5038 was observed in a search of molecules in a known DLA. The redshift path was included in our statistical sample to search for unknown sub-DLAs.

- QSO B0841+129 was observed in a study of the metal content of DLAs at $z_{\mathrm{abs}} \gtrsim 2$. The redshift path was included in the statistical sample. An additional sub-DLA reported by Fox et al. (2011) was excluded because it is within $3000 \mathrm{~km} \mathrm{~s}^{-1}$ of the quasar emission line.

- QSO B0908+0603 was observed in a study of the tomography of the IGM. The redshift path wss excluded from our statistical sample.

- QSO B0913+0715 was targeted with the aim of measuring the metal content and to search for deuterium in a known DLA. Therefore, the redshift path was included in our statistical sample to search for unknown sub-DLAs.

- QSO B0919-260 was targeted in a study of the metal content of $\mathrm{Mg}$ II absorbers at $0.6<z<1.5$. The redshift path was included to search for sub-DLAs at higher redshift.

- QSOB0933-333 was observed with the aim of measuring the metal content of a known DLA. The redshift path was included in our statistical sample to search for unknown sub-DLAs.

- QSO B0951-0405 was targeted with the aim of measuring the metal content of known DLAs. The redshift path and an additional sub-DLA reported by Guimarães et al. (2009) were included in the statistical sample.

- QSO B0952-0115 was first observed in a study of the tomography of the IGM and later to measure the metal content of a known DLA. To be conservative, the redshift path and an additional sub-DLA reported in Paper I were excluded from our statistical sample.

- QSO B1005-333 was targeted in a study of the metal content of $\mathrm{Mg}$ II absorbers at $0.6<z<1.5$. The redshift path was included to search for sub-DLAs at higher redshift.

- QSO B1027-0540 was observed in a study of the epoch of reionization. The redshift path was excluded because the quasar is $z_{\mathrm{em}}>5.0$.

- Q 1036-272 was observed to constrain the variation of ratio of the electron to proton masses. The redshift path was included in the statistical sample to search for unknown sub-DLAs. 
- QSOB1036-2257 was targeted with the aim of measuring the metal content of a known DLA. Therefore, the redshift path and an additional sub-DLA reported in Paper I were included in our statistical sample.

- QSO J1039-2719 was observed to constrain the variation of ratio of the electron to proton masses in a known sub-DLA and in a study of narrow absorption lines associated with the quasar. The redshift path was included in the statistical sample to search for additional sub-DLAs.

- QSO B1038-2712 was observed in Visitor Mode as part of a program to search for molecules in known DLAs but the purpose of observing this particular target was to confirm the BAL nature of the quasar. Hence, the redshift path was included in our statistical sample.

- QSOB1036-268 was targeted in a study of the BAL outflow of the quasar. Hence, the redshift path and an additional sub-DLA reported in Paper I were included in our statistical sample.

- J104642.9+053107 was observed in a study of the tomography of the IGM. Therefore, the redshift path was excluded from the statistical sample.

- QSO B1055-301 was observed with the aim of measuring the metal content of a known DLA. Therefore, the redshift path was included in our statistical sample to search for unknown sub-DLAs.

- QSO B1101-26 was targeted in a study of the variation of the fine-structure constant in a quasar with known metal lines. Hence, the redshift path was excluded from the statistical sample.

- QSO B1104-181 was observed in a study of the tomography of the IGM. The redshift path was excluded from our statistical sample.

- QSO B1108-07 was targeted with the aim of measuring the metal content and search for deuterium in a known DLA. Hence, the redshift path and an additional sub-DLA reported by Ledoux et al. (2006) were included in our statistical sample.

- QSO B1113-1533 was observed in a search of deuterium in a known DLA. The redshift path was included in our statistical sample to search for unknown sub-DLAs.

- QSO B1114-0822 was observed with the aim of measuring the metal content of a known DLA. The redshift path was included in our statistical sample to search for unknown sub-DLAs.

- QSO B1122-168 was targeted in a study of the high ionization metal lines in the IGM and broad-line regions. The redshift path was excluded from our statistical sample.

- QSO J1142+2654 was observed in a study of the He II reionization. The redshift path was excluded from our statistical sample.

- QSO B1151+068 was observed in a study of the metal content in a known DLA. The redshift path was included in our statistical sample to search for unknown sub-DLAs.

- J115538.6+053050 was observed in a study of the metal content of known DLAs. Therefore, the redshift path was included in our statistical sample to search for unknown sub-DLAs.

- QSO J1159+1337 was observed in a study of narrow absorption lines associated with the quasar. Therefore, the redshift path and an additional sub-DLA reported by Guimarães et al. (2009) were included in our statistical sample to search for unknown sub-DLAs.
- QSO B1158-1842 was targeted as part of the ESO Large Program for IGM studies. The redshift path was excluded from the statistical sample.

- QSO B1202-074 was observed under the quasar environments, ESO Large Program for IGM studies and DLA abundances programs. Therefore, the redshift path was not included in our statistical sample.

- J120550.2+020131 was observed to confirm the nature of a DLA candidate. The redshift path was included in our statistical sample to search for unknown sub-DLAs.

- QSO B1209+0919 was targeted with the aim of measuring the metal content of a known DLA. The redshift path was included in our statistical sample to search for unknown sub-DLAs.

- LBQS 1209+1046 was targeted with the aim of measuring the metal content of a known DLA. The redshift path was included in our statistical sample to search for unknown sub-DLAs.

- LBQS 1210+1731 was observed in a study of the metal content of a DLA at $z_{\mathrm{abs}} \approx 2$. The redshift path was included in the statistical sample to search for unknown sub-DLAs.

- QSO B1220-1800 was observed to confirm the nature of a sub-DLA candidate. The redshift path was included in our statistical sample to search for unknown sub-DLAs.

- LBQS 1223+1753 was observed in a study of the quasar broad-line region and metal content in a known DLA. The redshift path and an additional sub-DLA reported by Dessauges-Zavadsky et al. (2003) were included in our statistical sample.

- QSOB1228-113 was observed with the aim of measuring the metal content of known DLAs. The redshift path was included in our statistical sample to search for unknown sub-DLAs.

- QSO B1230-101 was targeted in a study of the metal content of $\mathrm{Mg}$ II absorbers at $0.6<z<1.5$. The redshift path was included to search for sub-DLAs at higher redshift.

- LBQS 1232+0815 was observed in a study of the molecular content of a known DLA, quasar outflows and to constrain the variation of ratio of the electron to proton masses. The redshift path and an additional sub-DLA reported in Paper I were included in the statistical sample.

- LBQS 1242+0006 was observed to confirm the nature of a DLA candidate. The redshift path was included in our statistical sample to search for unknown sub-DLAs.

- LBQS 1246-0217 was observed in a study of the metal content of a known DLA. The redshift path was included in our statistical sample to search for unknown sub-DLAs.

- J124957.2-015929 was observed in a study of the He II reionization. The redshift path was excluded from our statistical sample.

- QSO B1256-177 was targeted in a study of the metal content of $\mathrm{Mg}$ II absorbers at $0.6<z<1.5$. The redshift path was included to search for sub-DLAs at higher redshift.

- QSOB1306+0356 was observed in a study of the epoch of reionization. The redshift path was excluded because the quasar is $z_{\mathrm{em}}>5.0$.

- QSO B1317-0507 was observed in a study of the He II reionization. The redshift path was excluded from our statistical sample.

- QSO B1318-263 was targeted in a study of the metal content of $\mathrm{Mg}$ II absorbers at $0.6<z<1.5$. The redshift path was included to search for sub-DLAs at higher redshift. 
- QSO B1324-047 was targeted in a study of the metal content of $\mathrm{Mg}$ II absorbers at $0.6<z<1.5$. The redshift path was included to search for sub-DLAs at higher redshift.

- QSO J1330-2522 was observed in a study of the He II reionization. The redshift path and three sub-DLAs were excluded from our statistical sample.

- QSO B1331+170 was targeted in a study of the metal content in a known DLA. Hence, the redshift path was included in our statistical sample to search for known sub-DLAs.

- QSO J1344-1035 was targeted as part of the ESO Large Program for IGM studies. The redshift path was excluded from our statistical sample.

- QSO J1347-2457 was observed as part of the ESO Large Program for IGM studies. The redshift path was excluded from the statistical sample.

- QSO J1356-1101 was observed with the aim of measuring the metal content of known DLAs. Therefore, the redshift path and an additional sub-DLA reported in Paper I were included in our statistical sample.

- QSO B1402-012 was targeted in a study of the metal content of Mg II absorbers at $0.6<z<1.5$. The redshift path was included to search for sub-DLAs at higher redshift.

- QSO B1409+0930 was observed in a study of the metal content of known DLAs. The redshift path and an additional sub-DLA reported by Fox et al. (2009) were included in our statistical sample.

- QSO B1412-096 was targeted in a study of the metal content of $\mathrm{Mg}$ II absorbers at $0.6<z<1.5$. The redshift path was included to search for sub-DLAs at higher redshift.

- QSO J1421-0643 was observed with the aim of measuring the metal content of a known DLA. Therefore, the redshift path was included in our statistical sample to search for unknown sub-DLAs.

- QSO B1429-008B was targeted in a study of the tomography of the IGM. The redshift was excluded from our statistical sample.

- QSO J1439+1117 was observed with the aim of measuring the molecular content of a known sub-DLA. The redshift path was included in our statistical sample to search for unknown sub-DLAs.

- QSO J1443+2724 was targeted in a search of molecules and measure the CMB temperature in a known DLA. The redshift path was included in our statistical sample to search for unknown sub-DLAs.

- LBQS 1444+0126 was observed in a study of the quasar broad-line regions, as well as the metal and molecular content of a known DLA. The redshift path was included in our statistical sample to search for additional unknown sub-DLAs.

- QSOB1448-232 was targeted for the IGM ionization and ESO Large Program for IGM studies. The redshift path was excluded from our statistical sample.

- J145147.1-151220 was observed as part of the ESO Large Program for IGM studies. The redshift path was not included in our statistical sample.

- J151352.52+085555.7 was observed in a study of the quasar outflows. Therefore, the redshift path was included in our statistical sample to search for unknown sub-DLAs.

- QSO J1621-0042 was observed in a study of the He II reionization. The redshift path was excluded from our statistical sample.

- 4C 12.59 was observed in a study of the metal content of known low-redshift DLA and sub-DLA. The redshift path was included in our statistical sample to search for subDLAs at higher redshift.

- QSO J1723+2243 was observed in a search of molecules in a known DLA. The redshift path and an additional sub-DLA reported in Paper I were included in our statistical sample.

- QSO B1937-1009 was observed in a study of the He II reionization. The redshift path was excluded from our statistical sample.

- QSO B1935-692 was observed in a study of the He II reionization. The redshift path was excluded from our statistical sample.

- QSO B2000-330 was observed as part of the ESO Large Program for IGM studies and in a study of Jeans scale of the IGM. The redshift path and sub-DLAs reported by O'Meara et al. (2007) were not included in the statistical sample.

- LBQS 2113-4345 was targeted in a study of the tomography of the IGM. The redshift was excluded from our statistical sample.

- LBQS 2114-4347 was targeted in a study of the tomography of the IGM. The redshift and a sub-DLA reported in Paper I were excluded from our statistical sample.

- J211739.5-433538 was targeted in a study of the tomography of the IGM. The redshift was excluded from our statistical sample.

- QSO J2119-3536 was targeted in a study of the metal content of a known sub-DLA. Therefore, the redshift path was included in our statistical sample to search for unknown sub-DLAs.

- QSO B2126-15 was observed as part of the ESO Large Program for IGM studies. The redshift path and two subDLAs reported by O'Meara et al. (2007) were not included in our statistical sample.

- QSO B2129-4653 was targeted in a study of the variation of the fine-structure constant in a quasar with known metal lines. Hence, the redshift path was excluded from the statistical sample.

- J213314.2-464031 was observed in Visitor Mode as part of a program to search for molecules in known DLAs but the purpose of observing this particular target was to study the tomography of the IGM (see Coppolani et al. 2006). Hence, the redshift path was excluded from our statistical sample.

- LBQS 2132-4321 was targeted in a study of the metal content of a known sub-DLA. The redshift path was included in our statistical sample to search for unknown sub-DLAs.

- LBQS 2138-4427 was observed with the aim of measuring the metal content of known DLAs. The redshift path was included in our statistical sample to search for unknown sub-DLAs.

- QSO B2139-4433 was observed in a study of the tomography of the IGM. The redshift path was excluded from our statistical sample.

- QSO B2149-306 was targeted in a study of the metal content of $\mathrm{Mg}$ II absorbers at $0.6<z<1.5$. The redshift path was included to search for sub-DLAs at higher redshift.

- QSO B2204-408 was observed in a study of absorption lines associated with the quasar. Therefore, the redshift path was included in our statistical sample to search for unknown sub-DLAs.

- LBQS 2206-1958A was targeted in a study of the metal and molecular content of known DLAs. Therefore, the redshift path was included in our statistical sample to search for unknown sub-DLAs.

- QSO J2220-2803 was observed as part of the ESO Large Program for IGM studies and in a study of broad-line re- 
gions of the quasar. The redshift path was not included in our statistical sample.

- QSO B2222-396 was observed with the aim of measuring the metal content of a known DLA. The redshift path was included in our statistical sample to search for unknown sub-DLAs.

- QSO J2227-2243 was observed in a study of the IGM. The redshift path was excluded from the statistical sample.

- QSO B2225-4025 was observed with the aim of measuring the metal content of a known DLA. The redshift path was included in our statistical sample to search for unknown sub-DLAs.

- LBQS 2230+0232 was observed in a study of the metal content of a DLA at $z_{\mathrm{abs}} \gtrsim 2$. The redshift path was included in the statistical sample to search for known sub-DLAs.

- J223851.0-295301 was targeted in a study of the tomography of the IGM. The redshift was excluded from our statistical sample.

- J223922.9-294947 was targeted in a study of the tomography of the IGM. The redshift was excluded from our statistical sample.

- J223928.9-295451 was targeted in a study of the tomography of the IGM. The redshift was excluded from our statistical sample.

- J223941.8-294955 was targeted in a study of the tomography of the IGM. The redshift and a sub-DLA reported in Paper I were excluded from our statistical sample.

- J223948.7-294748 was targeted in a study of the tomography of the IGM. The redshift was excluded from our statistical sample.

- J223951.9-294837 was targeted in a study of the tomography of the IGM. The redshift was excluded from our statistical sample.

- QSO B2237-0607 was targeted in a search of new subDLAs. The redshift path was included in the statistical sample.

- QSO J2247-1237 was targeted in a study of the metal content of $\mathrm{Mg}$ II absorbers at $0.6<z<1.5$. The redshift path was included to search for sub-DLAs at higher redshift.

- QSO B2311-373 was observed with the aim of measuring the metal content of a known DLA. Therefore, the redshift path was included in our statistical sample to search for unknown sub-DLAs.
- J232046.7-294406 was targeted in a study of the tomography of the IGM. The redshift was excluded from our statistical sample.

- J232059.4-295520 was targeted in a study of the tomography of the IGM. The redshift was excluded from our statistical sample.

- J232114.3-294725 was observed in a study of the tomography of the IGM. Therefore, the redshift path was excluded from the statistical sample.

- J232121.2-294350 was targeted in a study of the tomography of the IGM. The redshift was excluded from our statistical sample.

- QSO B2318-1107 was observed in a search of molecules and measure the metal content of known DLAs. The redshift path was included in our statistical sample to search for unknown sub-DLAs.

- QSO B2332-094 was targeted with the aim of measuring the metal and molecular content of a known DLA. The redshift path and an additional sub-DLA reported by Ledoux et al. (2003) were included in our statistical sample.

- QSO B2342+3417 was observed with the aim of measuring the metal content of a known DLA. The redshift path and an additional sub-DLA reported in Paper I were included in our statistical sample.

- QSO J2346+1247 was observed in a study of the absorbers associated with known galaxies along the quasar line of sight. The redshift path was excluded from our statistical sample.

- QSO B2343+125 was targeted in a study of the metal content of a known DLA. Hence, the redshift path was included in our statistical sample.

- QSO B2345+000 was observed in a study of the absorbers associated with known galaxies along the quasar sightline. The redshift path was excluded from our statistical sample.

- QSO B2347-4342 was observed as part of the ESO Large Program and other IGM related programmes. The redshift path was not included in the statistical sample.

- QSO B2348-147 was observed in a study of the metal content of a known DLA. The redshift path was included in our statistical sample to search for unknown sub-DLAs.

- J235702.5-004824 was observed in a study of its BAL features and therefore the redshift path was included in our statistical sample to search for unknown sub-DLAs. 